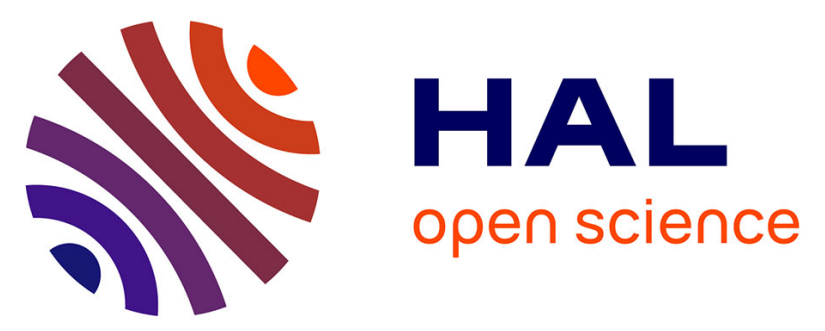

\title{
Box tomography: localized imaging of remote targets buried in an unknown medium, a step forward for understanding key structures in the deep Earth
}

Yder J. Masson, Barbara Romanowicz

\section{- To cite this version:}

Yder J. Masson, Barbara Romanowicz. Box tomography: localized imaging of remote targets buried in an unknown medium, a step forward for understanding key structures in the deep Earth. Geophysical Journal International, 2017, 211 (1), pp.141 - 163. 10.1093/gji/ggx141 . hal-01653929

\section{HAL Id: hal-01653929 \\ https://hal.science/hal-01653929}

Submitted on 2 Dec 2017

HAL is a multi-disciplinary open access archive for the deposit and dissemination of scientific research documents, whether they are published or not. The documents may come from teaching and research institutions in France or abroad, or from public or private research centers.
L'archive ouverte pluridisciplinaire HAL, est destinée au dépôt et à la diffusion de documents scientifiques de niveau recherche, publiés ou non, émanant des établissements d'enseignement et de recherche français ou étrangers, des laboratoires publics ou privés. 


\title{
Box tomography: localized imaging of remote targets buried in an unknown medium, a step forward for understanding key structures in the deep Earth
}

\author{
Yder Masson ${ }^{1}$ and Barbara Romanowicz ${ }^{1,2,3}$ \\ ${ }^{1}$ Institut de Physique du Globe, 1 Rue Jussieu, F-75005 Paris, France.E-mail: yder.masson@gmail.com \\ ${ }^{2}$ Collège de France, 11 place Marcelin Berthelot, F-75231 Paris Cedex 05, Paris, France \\ ${ }^{3}$ Berkeley Seismological Laboratory, University of California, Berkeley, CA 94720, USA
}

Accepted 2017 April 12. Received 2017 March 30; in original form 2016 October 18

\begin{abstract}
SUMMAR Y
We discuss an application of tomographic methods to the imaging of localized geological structures buried inside the Earth, where neither seismic sources nor receivers are necessarily present. We call this 'box tomography'. The essential difference between box-tomography and standard tomographic methods is that the numerical modelling (i.e. the ray tracing in traveltime tomography and the wave propagation in waveform tomography or full waveform inversion) is completely confined within the small box-region imaged. Thus, box tomography is a lot more efficient than global tomography (i.e. where we invert for the velocity in the larger volume that encompasses all the sources and receivers), for imaging localized objects. The principal difficulty in the box tomography problem comes from the medium outside the box-region imaged, that masks the target structure. In the data recorded at the surface, the information carried by the waves about the target structure is mixed with that about the surrounding medium. The main focus of this paper is to evaluate how well box tomography can extract useful information to produce accurate images of remote buried objects, especially in the difficult situation where the velocity distribution above the target structure is not known a priori. We implemented traveltime box tomography, where first arrival times are inverted using ray theory, and, waveform box tomography or full waveform box inversion, where seismograms are inverted using either scattering integrals or adjoint methods. Whatever the method employed, we found that, in the simple situation where the velocity structure is known around the target region, box-tomography is faster and more accurate than global tomography. Most importantly, through examples and simple analysis, we show that box-tomography can produce accurate images of localized structures even though the velocity distribution is not $a$ priori known around the box-region imaged. In the last section of this paper, we evaluate the usage of box-tomography for imaging geological structures in the deep Earth. We performed 2-D synthetic experiments in a fluid planet and we argue that box tomography can be employed for refining our current global tomographic models within arbitrary regions inside the Earth. This has important implications for understanding the composition and dynamics of the Earth's interior.
\end{abstract}

Key words: Interferometry; Inverse theory; Numerical solutions; Seismic tomography; Theoretical seismology; Wave scattering and diffraction.

\section{INTRODUCTION}

The imaging of remote geological structures buried inside the Earth is a subject of important research effort in seismology and exploration geophysics.

Seismology concentrates on targets with sizes ranging from tens of kilometres to the planet's dimension. Since the introduction of the Preliminary Reference Earth Model (Dziewonski \& Anderson 1981), many generations of global tomographic models have been developed and give us access to the 3-D struc- ture of the Earth's interior (e.g. Woodhouse \& Dziewonski 1984; Su \& Dziewonski 1993; Mégnin \& Romanowicz 2000; Houser et al. 2008; Kustowski et al. 2008; Simmons et al. 2010; Ritsema et al. 2011; Chang et al. 2015). The latest generation of global tomographic models (e.g. Lekić \& Romanowicz 2011; French et al. 2013; French \& Romanowicz 2014; Bozdağ et al. 2016) has emerged with the development of accurate numerical wavefield computations in a 3-D earth combined with access to enhanced High Performance Computer capabilities. These models have sharpened up mantle images and unveiled relatively 


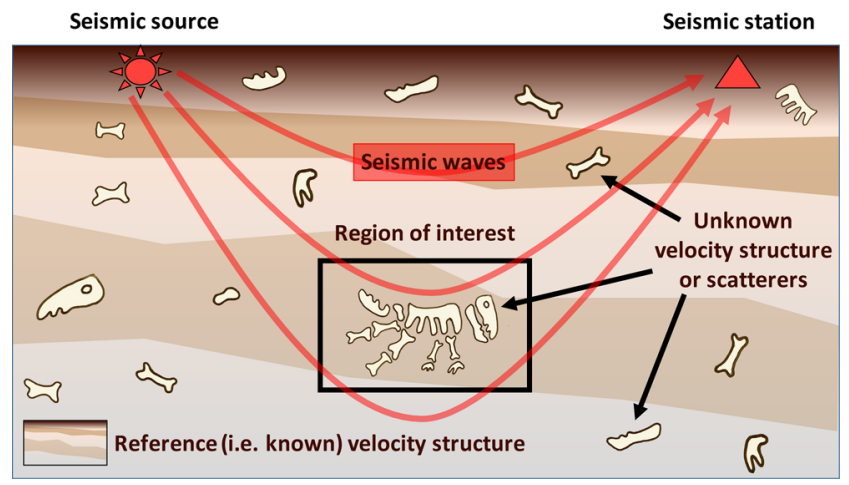

Figure 1. The box tomography problem. Seismic imaging or seismic tomography aims at constructing maps of underground structures. The geological medium is illuminated by artificial (e.g. seismic vibrator trucks, explosions) or natural (e.g. earthquakes) seismic sources that generate waves. As they propagate, these seismic waves (sketched in red) will interact with objects or structures (represented by bones) present along their path and accumulate information about the propagating medium. To collect this information, one records the waves at seismic stations, usually located near the surface of the Earth. The imaging process consists of transforming or inverting the seismic data recorded (e.g. traveltimes, waveforms or seismograms) into maps of the seismic velocity or of other physical properties. Box tomography aims at imaging only a small region or box (delimited by the black rectangle) that contains some objects of interest (the dinosaur skeleton). In this case, the difficulty comes from the fact that seismic waves propagate in the entire volume between the seismic source and the seismic station and are affected by both the objects inside (dinosaur skeleton) and the objects outside (scattered bones) the box of interest. Therefore, the useful information about the object of interest is mixed with irrelevant information about the surrounding structure in the recorded data. Our objective in this paper is to verify that it is possible to extract the useful information contained in the seismic data and produce detailed images of remote objects or targets with no (or limited) prior knowledge about the structure in the region surrounding the imaging box.

small scale structures that were blurred out in previous generation models. Finger-like structures have been found at the base of the oceanic asthenosphere (i.e. French et al. 2013), and vertically oriented broad low velocity plume conduits extend throughout the lower mantle beneath those major hotspots that are located within the perimeter of the deep mantle large low shear velocity provinces. While providing new insights into our understanding of mantle dynamics, the detailed morphology of these features requires further efforts to obtain higher resolution images.

Exploration geophysics (e.g. Burger 1992; Virieux \& Operto 2009) concentrates on the Earth's subsurface (say the first few kilometres) and aims at characterizing, among others, reservoirs of hydrocarbons, water or minerals, as well as monitoring their evolution over time. Because the majority of the easily accessible oil that is located at shallower depths has been discovered and is currently being produced, future reservoir discoveries will depend on our ability to develop efficient imaging methods aimed at deeper geological environments such as subsalt (e.g. Farmer et al. 1994) or deep water regions.

In this paper, we discuss what we call the 'box tomography' problem, that is the imaging of localized target structures buried in an unknown medium as illustrated in Fig. 1. A frequently encountered situation in geophysical imaging is that, once we have obtained an image of a given region in the Earth's interior, we discover new geological structures that attract our attention. This often leads us to reprocess our data in order to refine the original image in the vicinity of these newly found target structures, in order to gather more geological information. In order to construct the refined image, we typically have the following information available: First, a coarse (i.e. with a limited resolution) image (e.g. a velocity model) of the geological medium in the volume surrounding the target of interest and encompassing the sources and the receivers (represented by the layered medium in Fig. 1); second, the approximate location and dimensions of the target of interest (as represented by the black rectangle in Fig. 1); third, the data set (e.g. traveltimes, seismograms or waveforms) that was used to construct the original image, which can eventually be enriched with new data that are sensitive to the target region (e.g. associated with the red wave paths that pass through the box in Fig. 1). The lacking information in the box tomography problem is the fine scale structure inside and outside the box containing the target of interest (as represented by the bones in Fig. 1). In the situation where both the seismic sources and the receivers are located far away from the target, the seismic data are likely equally sensitive to (i.e. they get equally perturbed by) the fine scale structure inside and outside the imaging box. The main focus of this paper is to verify whether or not we can resolve the fine scale structure within the imaging box with no prior knowledge about the fine scale structure of the propagating medium outside the imaging box.

The global refinement of the original image (i.e. where we try to improve the resolution everywhere and not only around the target) is often impossible, in many cases simply because we already reached the maximum resolution achievable with the computational power available. Short of being able to do that, there are essentially two strategies for target-oriented seismic imaging.

A first approach generally named 'redatuming' consists of extrapolating the seismic data recorded at the surface of the Earth thanks to dense receiver arrays, to the surface of the imaging box. This way we obtain a new virtual data set where the sources and receivers have been moved to the vicinity of the target region. Then, the virtual data set is used along with standard imaging techniques such as time reverse migration (e.g. Baysal et al. 1983; Whitmore 1983; Biondi \& Shan 2002; Yoon et al. 2003, 2004; Mulder \& Plessix 2004; Bednar et al. 2006) or adjoint methods (e.g. Bamberger et al. 1982; Tarantola 1984, 1988; Tromp et al. 2005; Fichtner et al. 2006; Plessix 2006; Chen 2011) to image the region of interest. The advantage of redatuming is that, once the virtual data set is available, wave propagation only needs to be carried out locally within the small target volume, which greatly reduces the computational cost of the imaging process. Different approaches have been proposed for redatuming, for example, time reversal (e.g. Berryhill 1984), interferometry (e.g. Wapenaar et al. 2008; Schuster 2009), or more recently Marchenko redatuming or autofocusing (e.g. Rose 2002; Broggini et al. 2012; Wapenaar et al. 2013; Costa Filho et al. 2014; Wapenaar 2014). Though early redatuming methods based on time-reversal require a detailed velocity model of the medium above the target region to give accurate results, newer algorithms based on Marchenko redatuming work well when only a smooth estimate of the propagation velocity model is available. In general, redatuming methods require a dense sampling of the wavefield along a surface (e.g. the free surface of the Earth) to accurately extrapolate the seismic wavefield at depth. In exploration geophysics this is usually not problematic, as the acquisition setup can be constructed according to the imaging method employed (i.e. one can somehow choose the number, spacing and location of the seismic sources and receivers). In global seismology, however, the sources illuminating the Earth's interior are earthquakes that have an uneven geographic distribution (i.e. they are mostly located at the boundaries of tectonic plates), further, the majority of seismic 
stations are located on land and very few data are available in the oceans that cover most of the Earth's surface, and especially in the southern hemisphere. Because of this, and even though the global coverage of seismic stations has improved over the years, the use of redatuming techniques for imaging remote targets in the deep Earth remains challenging.

In this paper, we investigate an alternative approach to redatuming that we specifically refer to as box tomography. When working with ray theory there is no real difference between redatuming and box-tomography and the remaining part of this introduction mostly concerns waveform box tomography or full waveform box inversions, where the data set employed consists of entire seismograms. Similarly to redatuming, box tomography relies on local wave propagation modelling (i.e. where the wave propagation modelling is confined within the imaging box) combined with wavefield extrapolation for computational efficiency. There are however essential differences between the two approaches that we now summarize.

First, where redatuming restricts the computation to a finite domain (enclosing the virtual sources and receivers) using absorbing boundary conditions, box tomography employs dedicated boundary conditions to confine the wave propagation modelling within the imaging box, that is, to reproduce the exact same wavefield as the one that would be obtained when modelling wave propagation in the larger volume encompassing the sources and the receivers. This can be done, for example, using hybrid modelling methods where exact wave-propagation modelling is used inside the imaged volume and coupled with fast approximate solutions for modelling wave propagation outside that volume (see e.g. Bielak \& Christiano 1984; Moczo et al. 1997; Wen \& Helmberger 1998; Bielak et al. 2003; Capdeville et al. 2003; Yoshimura et al. 2003; To et al. 2005; Krishnan et al. 2006; Bouchon \& Sánchez-Sesma 2007; Zhao et al. 2008; Godinho et al. 2009; Opršal et al. 2009; Roecker et al. 2010; Monteiller et al. 2013; Pageot et al. 2013; Tong et al. 2014a,b). In this study, we take advantage of the most recent developments in injection methods (e.g. Alterman \& Karal 1968; Taflove \& Brodwin 1975; Robertsson \& Chapman 2000; Robertsson et al. 2000; Van Manen et al. 2007; Masson et al. 2014; Masson \& Romanowicz 2017). The principle of injection methods is to first model wave propagation through a reference velocity model in the global volume that encompasses all the sources and receivers. During this operation, the so-called reference wavefield is recorded at the surface of a smaller sub-volume, that is, the box region. In subsequent simulations, the wavefield is modelled locally thanks to virtual sources constructed from the reference wavefield and placed at the surface of the box region. The considerable advantage of this approach is that many small scale simulations can be performed to obtain the exact response induced by arbitrary velocity perturbations inside the imaging box and thus permit fast imaging.

Second, in contrast to redatuming, box-tomography does not use a two-step procedure (i.e. where one first extrapolates the data and then performs localized imaging) but integrates the wavefield extrapolation step into the wave propagation modelling. This can be done by solving the so-called 'scattering problem' numerically as proposed by Masson \& Romanowicz (2017).

Third, the wavefield that is extrapolated, the direction in which this wavefield is extrapolated, and the locations at which synthetic seismograms are computed differ. In redatuming, the observed wavefield is extrapolated downward (i.e. from the Earth's surface to the target region) and the misfit functional (that measures the difference between the observed and the synthetic seismograms) is evaluated at depth (i.e. at the locations of the virtual receivers).
Box tomography employs upward redatuming where the synthetic wavefield modelled within the imaging box is extrapolated upward, that is, from the surface of the imaging box to the receivers at the surface of the Earth. Thus the misfit functional is evaluated at the true locations of the seismic stations, exactly as in a global inversion where the entire volume encompassing the sources and the stations is imaged. The advantage of using upward redatuming is that the synthetic wavefield that is computed locally is completely known at the surface of the imaging box, therefore, wavefield extrapolation can be achieved exactly using classic representation theorems (e.g. De Hoop 1958; Aki \& Richards 1980; Snieder 2002; Masson et al. 2014). Thus, as opposed to redatuming, box-tomography does not rely on a dense sampling of the wavefield at the surface of the Earth to evaluate the misfit function and works with an arbitrary number of seismic stations or receivers.

Though all the methodological developments needed for doing box-tomography are available (see Masson et al. 2014; Masson \& Romanowicz 2017), their application to imaging problems is limited to a few studies (i.e. Roecker et al. 2010; Pageot et al. 2013; Monteiller et al. 2013; Tong et al. 2014a,b; Borisov et al. 2015; Monteiller et al. 2015; Willemsen et al. 2016). The main objective of this paper is to evaluate the performance of box-tomography for imaging localized structures in the difficult situation where the velocity distribution of the medium surrounding the imaging box is not known a priori.

In Section 2 of this paper, we review how different tomographic methods adapt to the box problem. Through numerical examples and simple analysis, we show that, when enough data is available, traveltime box tomography and waveform box tomography do not require complete knowledge of the velocity structure around the imaging box to produce accurate images of buried targets. In Section 3 of this paper, we investigate the possible use of box-tomography to image key geological structures in the deep Earth.

\section{BASIC CONCEPTS AND SIMPLE TESTS}

In this section, we first review traveltime tomography, which makes concepts easier to grasp, and its application to the box problem. Then, we expand our discussion to the case of waveform tomography. Our objective is to convince the reader that, under reasonable assumptions, box tomography can in theory produce accurate images of localized objects buried inside a medium with unknown velocity distribution.

\subsection{Localized imaging using traveltime tomography}

Traveltime tomography maps the slowness (i.e. the inverse of the velocity) from first arrival traveltimes measured between sources and receivers with known locations. For a given source/receiver pair (e.g. points A and D in Fig. 2a), the ray path $P$ (e.g. the solid black line in Fig. 2a) is prescribed by Fermat's principle (e.g. Born $\&$ Wolf 1959). Knowing the $i$ th ray path $P_{i}$, the traveltime $t_{i}$ needed by the waves to go from the source to the receiver is given by the path integral:

$t_{i}=\int_{P_{i}} s(\mathbf{x}) \mathrm{d} l \approx \sum_{j} l_{i j} s_{j}$,

where $s(\mathbf{x})$ denotes the slowness, $s_{j}$ is the slowness in the $j$ th cell of the slowness map (e.g. represented by grey boxes in Fig. 2a), $l_{i j}$ is the length of the $i$ th ray path through the $j$ th cell. For a problem 
(a) a global ray path

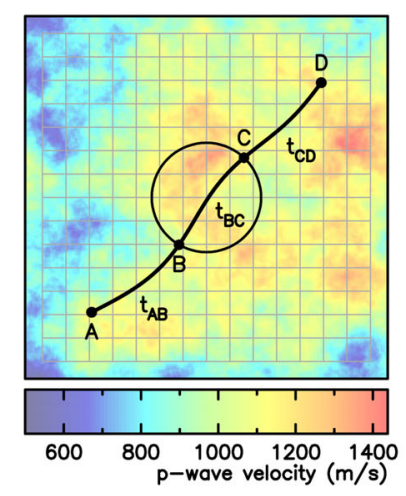

(c) a global wavefield

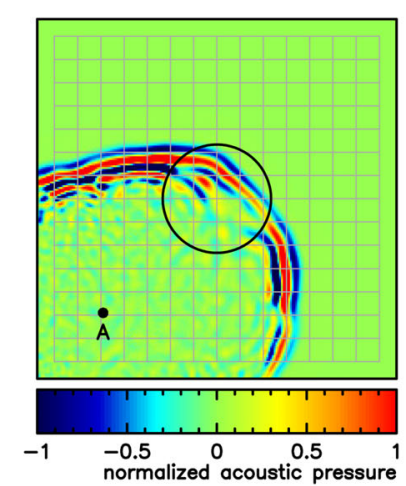

(e) a global sensitivity
kernel

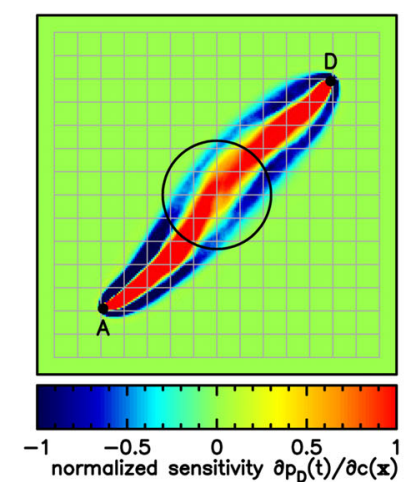

\section{(b) a local ray path}

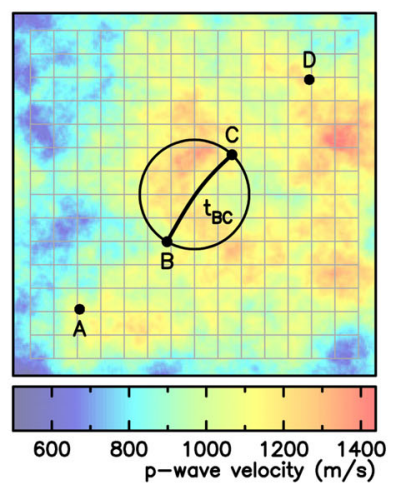

(d) a local wavefield

(e.g. Masson et al, 2013)

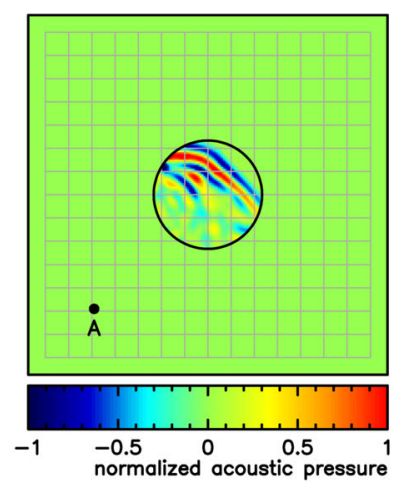

\section{(f) a local sensitivity kernel (e.g. Masson and Romanowicz, 2016)}

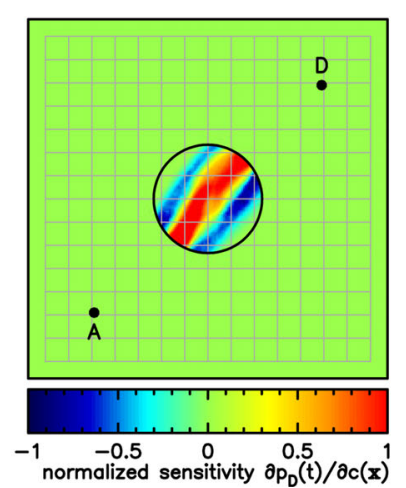

Figure 2. Illustration of some mathematical objects employed in classic tomography (left panels) and their local counterpart employed in box-tomography (right panels). (a) Minimum traveltime ray path between points A and D, among all possible paths linking point A to point D the solid black line represents the one for which the time $t_{i}$ in eq. (1) is minimum given the background velocity distribution $c(\mathbf{x})=1 / s(\mathbf{x})$ in colours. (b) Minimum traveltime ray path between points $\mathrm{B}$ and $\mathrm{C}$ that are the points where the global ray path in (a) intersects with the imaging box's surface represented by the circle. The global and the local ray paths are the same within the imaging box. (c) A global wavefield, that is, a snapshot of the acoustic pressure showing the waves generated by an explosive source in A at a given time $t$. (d) A local wavefield, that is the local counterpart of the global wavefield in (c), this wavefield is the same as the global wavefield within the circular imaging box and equal to 0 elsewhere. It is generated by virtual sources placed at the surface of the imaging box as detailed in Masson et al. (2014). (e) A global sensitivity kernel that quantifies how the wavefield $p(\mathbf{x}, t)$ recorded with a receiver in $\mathrm{D}$ at time a given $t$ and generated by an impulsive source in A varies when one changes the velocity $c(\mathbf{x})$ at location $\mathbf{x}$, two global simulations similar to the one in (c) are needed to evaluate the sensitivity kernel with eq. (22), a first simulation with an impulsive source (i.e. that has value 1 at time step 1 and value zero at later time steps) placed in A and a second simulation with an impulsive source placed in D. (f) A local sensitivity kernel, that is the local counterpart of the global sensitivity kernel in (e). This sensitivity kernel is the same as the global one within the circular imaging box and is equal to 0 elsewhere. It can be obtained by running two local simulations similar to the one in (d) as detailed in Masson \& Romanowicz (2017). 
involving $M$ traveltimes/ray paths and $N$ cells with unknown slownesses, eq. (1) gives the linear system:

$\mathbf{A m}=\mathbf{d}$

where $\mathbf{A}$ is an $M \times N$ matrix containing the ray lengths $l_{i j}$, $\mathbf{m}^{T}=\left(s_{1}, s_{2}, \ldots, s_{N}\right)$ contains the unknown model parameters or slownesses and $\mathbf{d}^{T}=\left(t_{1}, t_{2}, \ldots, t_{M}\right)$ contains the data, that is, the measured traveltimes. In real applications, the linear system in eq. (2) is usually ill posed and needs to be regularized to be solved. In this section however, for the sake of clarity, we will leave out the regularization problem that is not specific to box-tomography. We will use dense enough data sets so that the linear system in eq. (2) has an acceptable solution. In the situation where the ray paths are known (i.e. A is known), the tomographic problem is linear and the slowness map $\mathbf{m}$ can be directly obtained by solving the system in eq. (2), for example using a generalized least squares solution (Tarantola \& Valette 1982). In reality, however, the ray paths are not known a priori as they depend on the slowness $s(\mathbf{x})$. In this case, the tomographic problem becomes nonlinear and is solved iteratively. One usually starts with an a priori reference slowness model $\mathbf{m}_{0}=\mathbf{m}_{\text {ref }}$ that is used to estimate the ray paths, that is, to construct $\mathbf{A}_{0}=\mathbf{A}_{\text {ref }}$ and to compute synthetic traveltimes $\mathbf{d}_{0}=\mathbf{d}_{\text {ref }}$. At each iteration $n$, the model $\mathbf{m}_{n}$ is updated by inverting the residual traveltimes $\left(\mathbf{d}-\mathbf{d}_{n}\right)$ computed in the current model to obtain the model perturbation $\left(\mathbf{m}_{n+1}-\mathbf{m}_{n}\right)$, that is, by solving the linear system:

$\mathbf{A}_{n}\left(\mathbf{m}_{n+1}-\mathbf{m}_{n}\right)=\left(\mathbf{d}-\mathbf{d}_{n}\right)$

to obtain the updated slowness model

$\mathbf{m}_{n+1}=\mathbf{m}_{n}+\left[\mathbf{A}_{n}^{T} \mathbf{A}_{n}\right]^{-1} \mathbf{A}_{n}^{T}\left(\mathbf{d}-\mathbf{d}_{n}\right)$.

Once the new model $\mathbf{m}_{n+1}$ is available, it is used to recompute the synthetic traveltimes $\mathbf{d}_{n+1}$ and the ray path sensitivity matrix $\mathbf{A}_{n+1}$. The operation is repeated until the model $\mathbf{m}_{n+1}$ converges, for example, when the ray paths do not evolve anymore.

In box-tomography, we are only interested in mapping the slowness within a limited sub-volume that does not necessarily encompass the sources and the receivers, for example, within the small circle in Fig. 2. To address this problem, we can rewrite the linear system in eq. (2) as

$\mathbf{A} \mathbf{m}=\mathbf{A}^{\text {box }} \mathbf{m}^{\text {box }}+\mathbf{A}^{\text {ext }} \mathbf{m}^{\text {ext }}=\mathbf{d}$

where the vectors $\mathbf{m}^{\text {box }}$ and $\mathbf{m}^{\text {ext }}$ contain the slownesses of the cells inside and outside the imaging box, respectively. The matrices $\mathbf{A}^{\text {box }}$ and $\mathbf{A}^{\text {ext }}$ are reduced versions of $\mathbf{A}$ and contain information about the ray paths inside and outside the imaging box, respectively. In $\mathbf{A}^{\text {box }}$, all the columns corresponding to cells outside the imaging box are removed from $\mathbf{A}$. In $\mathbf{A}^{\mathrm{ext}}$, all the columns corresponding to cells inside the imaging box are removed from $\mathbf{A}$. In the situation where the ray paths and the slowness are known outside the imaging box eq. (5) transforms to

$\mathbf{A}^{\text {box }} \mathbf{m}^{\text {box }}=\mathbf{d}-\mathbf{A}^{\text {ext }} \mathbf{m}^{\text {ext }}=\mathbf{d}-\mathbf{d}^{\text {ext }}=\mathbf{d}^{\text {box }}$

where the vectors $\mathbf{d}^{\text {box }}$ and $\mathbf{d}^{\text {ext }}$ contain the actual traveltimes (i.e. in the true medium) inside and outside the imaging box, respectively. This corresponds to a classic local tomographic problem where the rays are anchored at their intersections with the imaging box boundary (e.g. at points B and C in Fig. 2b) and $\mathbf{d}^{\text {box }}$ is a redatumed data set obtained by correcting the traveltimes outside the imaging box (for example, we have $t_{B C}=t_{A D}-\left(t_{A B}+t_{C D}\right)$ in Fig. 2a). When the ray paths are not known a priori, the box tomography problem may be solved iteratively similarly to the standard problem.
By splitting the contribution of the cells inside and outside the imaging box, that is, using $\mathbf{A}_{n} \mathbf{m}_{n+1}=\mathbf{A}_{n}^{\text {box }} \mathbf{m}_{n+1}^{\text {box }}+\mathbf{A}_{n}^{\text {ext }} \mathbf{m}_{n+1}^{\text {ext }}$ and $\mathbf{A}_{n} \mathbf{m}_{n}=\mathbf{A}_{n}^{\text {box }} \mathbf{m}_{n}^{\text {box }}+\mathbf{A}_{n}^{\text {ext }} \mathbf{m}_{n}^{\text {ext }}$ in eq. (3) we obtain the linear system

$$
\begin{aligned}
\mathbf{A}_{n}^{\text {box }}\left(\mathbf{m}_{n+1}^{\text {box }}-\mathbf{m}_{n}^{\text {box }}\right) & =\left(\mathbf{d}^{\text {box }}-\mathbf{d}_{n}^{\text {box }}\right)+\left(\mathbf{d}^{\text {ext }}-\mathbf{d}_{n+1}^{\text {ext }}\right) \\
& =\Delta \mathbf{d}^{\text {box }}+\Delta \mathbf{d}^{\text {ext }}
\end{aligned}
$$

where $\mathbf{d}=\mathbf{d}^{\text {box }}+\mathbf{d}^{\text {ext }}$ contains the observed traveltimes and $\mathbf{d}^{\text {box }}$ and $\mathbf{d}^{\text {ext }}$ denote the traveltimes inside and outside the box in the real medium, respectively, $\mathbf{d}_{n}=\mathbf{d}_{n}^{\text {box }}+\mathbf{d}_{n}^{\text {ext }}$ contains the synthetic traveltimes computed in the model $\mathbf{m}_{n}$ and we have $\mathbf{d}_{n}=\mathbf{A}_{n} \mathbf{m}_{n}$, $\mathbf{d}_{n}^{\text {box }}=\mathbf{A}_{n}^{\text {box }} \mathbf{m}_{n}^{\text {box }}$ and $\mathbf{d}_{n}^{\text {ext }}=\mathbf{A}_{n}^{\text {ext }} \mathbf{m}_{n}^{\text {ext }}$, by definition, and, we introduced $\mathbf{d}_{n+1}^{\text {ext }}=\mathbf{A}_{n}^{\text {ext }} \mathbf{m}_{n+1}^{\text {ext }}$ that contains the synthetic traveltimes outside the imaging box computed in the updated model assuming that the ray paths remain unchanged. To obtain the box image, we thus evaluate

$\mathbf{m}_{n+1}^{\text {box }}=\mathbf{m}_{n}^{\text {box }}+\left[\left(\mathbf{A}_{n}^{\text {box }}\right)^{T} \mathbf{A}_{n}^{\text {box }}\right]^{-1}\left(\mathbf{A}_{n}^{\text {box }}\right)^{T}\left(\Delta \mathbf{d}^{\text {box }}+\Delta \mathbf{d}^{\text {ext }}\right)$

recursively until the box slowness model $\mathbf{m}_{n+1}^{\text {box }}$ converges. In theory, in order to update the slowness map $\mathbf{m}_{n}^{\text {box }}$ using eq. (8), we need $a$ priori knowledge about the updated slowness outside the imaging box $\mathbf{m}_{n+1}^{\text {ext }}$, because $\Delta \mathbf{d}^{\text {ext }}=\left(\mathbf{d}^{\text {ext }}-\mathbf{A}_{n}^{\text {ext }} \mathbf{m}_{n+1}^{\text {ext }}\right)$. In practice, however, it is a reasonable assumption to set $\mathbf{m}_{n}^{\text {ext }}=\mathbf{m}_{\text {ref }}^{\text {ext }} \forall n$ and to freeze the ray paths outside the imaging box by setting $\mathbf{A}_{n}^{\text {ext }}=\mathbf{A}_{\text {ref }}^{\text {ext }} \forall n$. This is because traveltimes are more sensitive to perturbations in the slowness than to perturbations in the ray paths, which makes it possible to image the slowness without knowing the exact ray paths (e.g. Snieder \& Sambridge 1992). Note that the ray paths in $\mathbf{A}^{\text {ext }}$ also depend on the slowness inside the imaging box, therefore, the starting model $\mathbf{m}_{0}=\mathbf{m}_{\text {ref }}$ should be close to the true slowness distribution. Freezing the velocity model outside the imaging box is the fundamental assumption in box-tomography. In the rest of this paper, we evaluate its consequences.

In the ideal situation where the slowness is known outside the imaging box, that is when $\mathbf{m}_{\mathrm{ref}}^{\text {ext }}=\mathbf{m}^{\text {ext }}$ contains the true slowness of the propagating medium, then $\Delta \mathbf{d}^{\text {ext }}$ vanishes in eq. (8) and the updated model $\mathbf{m}_{n+1}^{\text {box }}$ should converge to an accurate solution.

In the practical situation where the slowness is not known a priori outside the imaging box (i.e. we have $\Delta \mathbf{d}^{\text {ext }} \neq 0$ ), eq. (8) maps traveltimes that are sensitive to the slowness both inside and outside the imaging box to the local image $\mathbf{m}_{n+1}^{\text {box }}$ that is only defined within the imaging box. Therefore, our ability to produce an accurate slowness map within the imaging box depends on the extent to which the differential traveltimes $\Delta \mathbf{d}^{\text {ext }}$ induced by the unknown slowness outside the imaging box corrupt the local image. The differential traveltimes $\Delta \mathbf{d}^{\text {ext }}$ can be thought of as a noise which is added to the useful signal for retrieving information about the slowness structure inside the imaging box. Clearly, one cannot count on a high signal to noise ratio (i.e. for the amplitudes in $\Delta \mathbf{d}^{\text {ext }}$ to be much smaller than those in $\Delta \mathbf{d}^{\text {box }}$ ) because the unknown slowness anomaly likely has the same amplitude inside and outside the imaging box (if the error in the reference model $\mathbf{m}_{\text {ref }}$ is homogeneously distributed). In the worst case, where the reference medium $\mathbf{m}_{\mathrm{ref}}^{\text {ext }}$ is biased and does not explain the average traveltimes (i.e. we have a constant velocity offset outside the imaging box), then the times in $\Delta \mathbf{d}^{\text {ext }}$ are proportional to the length of the ray paths outside the imaging box and can be larger than those in $\Delta \mathbf{d}^{\text {box }}$. Fortunately, in tomography, such a systematic bias should be limited, this is because the reference model is usually a smoothed or averaged version of the true 
velocity distribution and does explain the average traveltimes. Thus we expect no systematic error in $\Delta \mathbf{d}^{\text {ext }}$ in practical applications.

The way the unwanted noise in $\Delta \mathbf{d}^{\text {ext }}=\left[\Delta t_{1}^{\text {ext }}, \ldots, \Delta t_{N_{i}^{\text {ext }}}^{\text {ext }}\right]$ depends on the distance travelled by the waves outside the box-region can be understood using basic statistics. Given that, the differential traveltime outside the imaging box

$\Delta t_{i}^{\mathrm{ext}}=\sum_{j=1}^{N_{i}^{\mathrm{ext}}} l_{i j} \Delta s_{j}^{\mathrm{ext}}$

is the sum of the differential traveltimes $\Delta t_{i j}^{\text {ext }}=l_{i j} \Delta s_{j}^{\text {ext }}$ within the $j=1, \ldots, N_{i}^{\text {ext }}$ out of the box cells along the ray path $P_{i}$, when the differential slowness $\Delta s_{j}^{\text {ext }}$ in the $j$ th out of the box cell is taken as a random variable, the variance of the total differential traveltime outside the imaging box is given by

$$
\begin{aligned}
\operatorname{Var}\left(\Delta t_{i}^{\mathrm{ext}}\right)= & \operatorname{Var}\left(\sum_{j=1}^{N_{i}^{\mathrm{ext}}} l_{i j} \Delta s_{j}^{\mathrm{ext}}\right) \\
= & \sum_{j=1}^{N_{i}^{\mathrm{ext}}} l_{i j}^{2} \operatorname{Var}\left(\Delta s_{j}^{\mathrm{ext}}\right) \\
& +2 \sum_{1 \leq j<k \leq N_{i}^{\mathrm{ext}}} l_{i j} l_{i k} \operatorname{Cov}\left(\Delta s_{j}^{\mathrm{ext}}, \Delta s_{k}\right) .
\end{aligned}
$$

In the situation where the differential slownesses $\Delta s_{j}^{\text {ext }}$ are pairwise independent (i.e. $\operatorname{Cov}\left(\Delta s_{j}^{\text {ext }}, \Delta s_{k}^{\text {ext }}\right)=0$ ) and the differential slowness, that is the error in the reference model $\mathbf{m}_{\text {ref }}$, is homogeneously distributed (i.e. we have $\operatorname{Var}\left(\Delta s_{j}^{\text {ext }}\right)=\operatorname{Var}\left(\Delta s^{\text {ext }}\right)=$ constant), taking $l_{i j} \approx l_{c}=$ constant where $l_{c}$ is the typical size of the cells, we obtain

$\operatorname{Var}\left(\Delta t_{i}^{\mathrm{ext}}\right)=l_{c}^{2} N_{i}^{\mathrm{ext}} \operatorname{Var}\left(\Delta s^{\mathrm{ext}}\right)$,

which shows that the magnitude of the noise $\Delta \mathbf{d}^{\text {ext }}$ induced by the residual slowness $\Delta s^{\text {ext }}$ outside the imaging box, as measured as its standard deviation

$\sigma\left(\Delta t_{i}^{\mathrm{ext}}\right)=\sqrt{\operatorname{Var}\left(\Delta \mathrm{t}_{\mathrm{i}}^{\mathrm{ext}}\right)}=l_{c} \sqrt{N_{i}^{\mathrm{ext}}} \sigma\left(\Delta s^{\mathrm{ext}}\right)$,

is proportional to the square root of the distance travelled by the waves outside the imaging box (i.e. to the square root of the number of cells $N_{i}^{\text {ext }}$ crossed by the rays). If the differential slownesses $\Delta s_{j}^{\text {ext }}$ are anti-correlated to some degree, the dependence of the differential traveltimes on the distance between the box and the sources/receivers may be smaller than expected, however, it has some implication regarding the residual slowness distribution. It is not impossible that such an anti-correlation exists when the starting or reference model is a smooth model obtained using a prior tomographic inversion with coarse resolution, because of the differential traveltime minimization (e.g. along ray sections that are poorly constrained, the inversion will add 'ghost' anti-correlated velocity perturbation to match the observed traveltimes). This is however a complicated problem that we will not discuss any further here.

In an attempt to quantify how the differential traveltimes in $\Delta \mathbf{d}^{\text {ext }}$ induced by the external structure affect the local image $\mathbf{m}_{n}^{\text {box }}$, we now consider the single cell box-tomography problem. In the situation where the imaging box contains a single cell with unknown slowness $s^{\text {box }}$, eq. (8) reduces to

$s_{n+1}^{\text {box }}=\left\langle s^{\text {box }}\right\rangle+\epsilon \approx s^{\text {box }}+\epsilon$ where

$$
\left\langle s^{\mathrm{box}}\right\rangle=s_{\mathrm{ref}}^{\mathrm{box}}+\frac{\sum_{i=1}^{M} l_{i}^{\mathrm{box}} \Delta t_{i}^{\mathrm{box}}}{\sum_{i=1}^{M}\left(l_{i}^{\mathrm{box}}\right)^{2}} \approx s^{\mathrm{box}}
$$

is an averaged value of the slowness within the imaging box and converges to the true slowness $s^{\text {box }}$ as the rays approach their true paths, and

$$
\epsilon=\frac{\sum_{i=1}^{M} l_{i}^{\mathrm{box}} \Delta t_{i}^{\mathrm{ext}}}{\sum_{i=1}^{M}\left(l_{i}^{\mathrm{box}}\right)^{2}}
$$

is the slowness error introduced by the unknown fraction of the slowness outside the imaging box. $l_{i}^{\text {box }}$ is the length of the $i$ th ray through the box cell, and, $\Delta t_{i}^{\text {box }}$ and $\Delta t_{i}^{\text {ext }}$ are the differences between the observed traveltimes and the computed traveltimes inside and outside the box cell, respectively. Taking $l_{i}^{\text {box }} \approx l_{i j} \approx l_{c}=$ constant, where $l_{c}$ is the typical size of the cells, and using $N_{i}^{\text {ext }} \approx N^{\text {ext }} \approx$ constant in eq. (9), eq. (15) becomes

$\epsilon \approx \frac{1}{l_{c}}\left\langle\Delta t^{\mathrm{ext}}\right\rangle \approx N^{\mathrm{ext}}\left\langle\Delta s^{\mathrm{ext}}\right\rangle$,

where $\left\langle\Delta t^{\mathrm{ext}}\right\rangle$ is the average of the differential traveltimes observed for the $M$ rays passing through the box cell and

$\left\langle\Delta s^{\mathrm{ext}}\right\rangle=\frac{1}{M N^{\mathrm{ext}}} \sum_{k=1}^{M N^{\mathrm{ext}}} \Delta s_{k}^{\mathrm{ext}}$

is the average of the differential slownesses of the $M N^{\text {ext }}$ cells crossed by the rays outside the imaging box. Eq. (16) shows that a necessary condition for obtaining an unbiased image of the slowness within a remote box region is that the average slowness anomaly $\left\langle\Delta s^{\text {ext }}\right\rangle$ vanishes outside the target region. In this case, the noise $\Delta \mathbf{d}^{\text {ext }}$ will map to the null space because the reference model explains the observed traveltimes on average. Fortunately, when starting with a model obtained from a prior inversion (e.g. performed in the total volume that encompasses the sources and the receivers), this condition is usually satisfied. This is because, tomographic inversions naturally recover a homogenized model of the true propagating medium (e.g. Capdeville et al. 2013). In other words, the reference model $s_{\text {ref }}(\mathbf{x})$ can be thought of as an averaged or smooth version of the actual medium $s(\mathbf{x})$, where the average is taken over a characteristic distance $l_{\text {res }}$ that more or less corresponds to the resolution length of the original image $s_{\text {ref }}(\mathbf{x})$. Exactly which average quantity is obtained when constructing the original model is a matter of debate as many homogenization, upscaling or effective medium theories coexist and give slightly different values for the effective moduli driving wave propagation at the wavelength scale. However, theoretical bounds exist (e.g. Hashin \& Shtrikman 1963) and simple averaging gives reasonable estimates (e.g. Masson \& Pride 2015). Therefore, when starting with a model obtained from a prior tomographic inversion with resolution length $l_{\text {res }}$, the average slowness anomaly $\left\langle\Delta s^{\mathrm{ext}}\right\rangle$ should indeed vanish, as long as the total distance sampled by the rays outside the imaging box is much larger than $l_{\text {res }}$. In this situation, box-tomography will give an accurate image of the target region.

To determine the rate at which the error $\epsilon$ in the local image degrades with the burial depth, one needs to quantify how the 
estimated mean $\left\langle\Delta s^{\text {ext }}\right\rangle$ in eq. (17) deviates from its true value $\left\langle\Delta s^{\text {ext }}\right\rangle_{\text {true }}=0$ when the distance between the imaging box and the sources/receivers is varying. This can be quantified using the standard error

$\operatorname{SE}\left\{\left\langle\Delta s^{\mathrm{ext}}\right\rangle\right\}=\frac{\sigma\left(\Delta s^{\mathrm{ext}}\right)}{\sqrt{M N^{\mathrm{ext}}}}$

that gives the typical error in the estimated mean $\left\langle\Delta s^{\text {ext }}\right\rangle$ as a function of the sample size $M N^{\text {ext }}$. It follows that the standard error of the mean $\epsilon$ in eq. (16) is given by

$\mathrm{SE}(\epsilon)=N^{\mathrm{ext}} \mathrm{SE}\left\{\left\langle\Delta s^{\mathrm{ext}}\right\rangle\right\}=\sqrt{\frac{N^{\mathrm{ext}}}{M}} \sigma\left(\Delta s^{\mathrm{ext}}\right)$.

Note the competing effects of the burial depth (which is proportional to $N^{\text {ext }}$ ) that is increasing the magnitude of the error $\epsilon$ and the size of the data set or the number of rays $M$ employed for the inversion that is reducing the magnitude of the error $\epsilon$. When the sample size is fixed, the typical error of the mean estimate $\epsilon$ in eq. (16) (i.e. its deviation from $\epsilon_{\text {true }}=0$ ) should not degrade faster than the square root of the typical distance between the box and the sources/receivers. Further, using large enough data sets (i.e. large enough $M$ ), we should be able to accurately image targets at arbitrary depth.

To illustrate our discussion, we now present practical examples. The objective in our tests is to image the subvolume or imaging box delimited by the small circle in Fig. 3 using traveltimes measured between distant sources and receivers located on the large circle. The target velocity model to be imaged, pictured in Fig. 3(a), is a self-affine random medium with Hurst exponent $H=-0.5$ (e.g. Klimeš 2002; Meschede \& Romanowicz 2015) and contains heterogeneities of all sizes. To construct the smooth starting model in Fig. 3(a), we filtered the target model in the Fourier domain by setting to zero all the Fourier coefficients with wavenumber $k=2 \pi / \lambda_{c}$ for which $\lambda_{c}<12.5 \mathrm{~cm}$. This means that the dimension of the imaging box in our problem is about twice the resolution of the reference or starting model. The unknown part of the velocity that we want to image is shown in Fig. 3(c). It is the difference between the target model in Fig. 3(a) and the reference model in Fig. 3(b). All models in Fig. 3 are defined on a regular grid with dimensions $100 \times 100$. To construct the data sets needed for the inversions, we traced rays between random pairs of points lying on the outer circle and we selected only the ray paths that go through the imaging box. For the ray tracing, we used the algorithm proposed by Prothero et al. (1988). A total number of 20000 rays have been selected for the inversions presented in Fig. 4. For each ray path $i$, we measured the total traveltime $t_{i}$, the traveltime inside the imaging box $t_{i}^{\text {box }}$ and the traveltime outside the imaging box $t_{i}^{\text {ext }}$, both for the target and reference models. These traveltimes were then assembled to obtain the residual traveltime vectors $\Delta \mathbf{d}^{\text {box }}$ and $\Delta \mathbf{d}^{\text {ext }}$ as defined in eq. (7). For the inverse problem, we used the same parametrization as for the models, that is, the entire slowness map is defined on a regular grid with dimensions $100 \times 100$ and a constant slowness is assigned to each cell. The unknowns of the tomographic problem are the $N_{\text {box }}=401$ slownesses of the cells located inside the imaging box, that is, inside the small circle in Figs 3 and 4.

In order to verify that imaging the box region within the small circle is possible without knowing the external velocity structure, we performed tomographic inversions with and without accounting for the traveltimes induced by the external structure. The results are presented in Fig. 4. The top panels show tomographic maps of the velocity within the imaging box (zoomed around the small circle in Fig. 3) and the bottom panels are scatter plots where the inverted velocity maps are compared to the actual velocity of the imaged medium. In all inversions, starting with the reference model $\mathbf{m}_{0}^{\text {box }}=\mathbf{m}_{\text {ref }}^{\text {box }}$ pictured in Fig. 3(b), we performed one box-tomography iteration by evaluating the right-hand side of eq. (8) to obtain the local image $\mathbf{m}_{1}^{\text {box }}$. Fig. 4(b) shows the image obtained by inverting the total traveltimes $\Delta \mathbf{d}^{\text {box }}+\Delta \mathbf{d}^{\text {ext }}$. This corresponds to the real world situation where the contributions from the internal and the external slowness structure add up and are not easily separable in the observed data. Fig. 4(c) shows the image obtained by inverting the internal traveltimes $\Delta \mathbf{d}^{\text {box }}$ (i.e. by setting $\Delta \mathbf{d}^{\text {ext }}=0$ in eq. (8)). This corresponds to the case where the slowness structure is fully known outside the imaging box. In this case the obtained image is the solution of a local tomographic problem performed with a redatumed data set (i.e. where the sources and the receivers are virtually moved onto the surface of the imaging box). Fig. 4(d), shows the difference between the image in Fig. 4(b) and the image in Fig. 4(c). Note that this is also the image that is obtained when inverting the external traveltimes (i.e. by setting $\Delta \mathbf{d}^{\text {box }}=0$ in eq. (8)), this is because the problem is linear and we perform a single iteration. This helps visualize how the uncertainty in the external slowness structure maps into the local image.

Not surprisingly, the local image obtained when the external velocity structure is known (in Fig. 4c) accurately reproduces the true velocity structure of the propagating medium. Looking at the scatter plot, we observe an excellent correlation between the inverted and the true velocities within the imaging box. We calculated the correlation coefficient $r=0.98$ that is very close to 1 which corresponds to a perfect correlation. Note that the distribution in the scatter plot is slightly rotated clockwise (i.e. the red solid line is rotated with respect to the blue dashed identity line $y=x$ ) which means that the absolute velocities are marginally underestimated in the box image.

The box image obtained by inverting the total traveltimes (in Fig. 4b) is also the sum of the images in Figs 4(c) and (d). Though it is a bit noisier, the visual aspect of the velocity map is still excellent when compared to the actual medium. The distribution in the scatter plot is more spread around the $y=x$ identity line compared to the model in Fig. 4(c) but the correlation coefficient $r=0.87$ is still very high. As in the previous case, the absolute velocities are marginally underestimated (i.e. the red solid line is rotated with respect to the blue dashed identity line $y=x$ ). The overall quality of the box-tomography model is remarkable given that the waves travel distances, in the unknown outer medium, that are four times longer than the radius of the box.

The bottom panels in Figs 4(b) and (c) shows the distribution of the error in the models (measured as the difference between the true velocity and the inverted velocity). The standard deviation $\sigma=0.41$ measured for the model obtained by inverting the total traveltimes (in Fig. 4b) is about twice that of the model obtain by ignoring the residual traveltimes induced by the external structure $\sigma=0.19$ (in Fig. 4c). As a comparison, the standard deviation of the true velocity anomaly map (in Fig. 4 a bottom panel) is $\sigma=0.83$.

The difference between the images obtained by taking (Fig. 4c) and not taking (Fig. 4d) into account the differential traveltimes induced by the external structure has small velocity anomaly amplitude which confirms that, provided that the external slowness distribution has zero mean and is properly sampled by the rays, the uncertainty in the external velocity model does not map to the box image. Further, looking at the scatter plot, we observe no correlation between the residual error and the actual velocity structure of the propagating medium. We estimated the correlation coefficient $r=0.08$ that is very close to 0 which is characteristic of 
(a)

\section{Complete velocity structure}
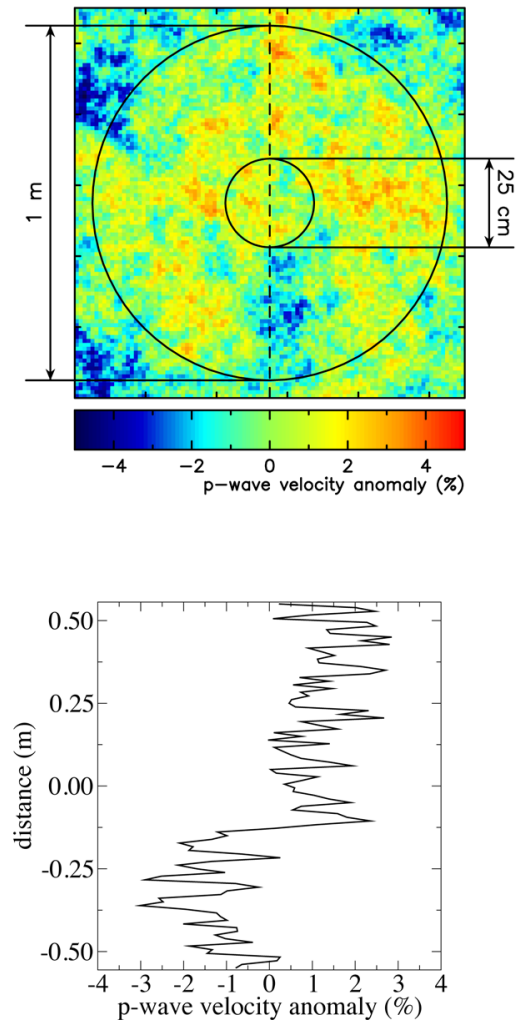

(b)

\section{Large scale velocity structure}

(that is known a-priori)

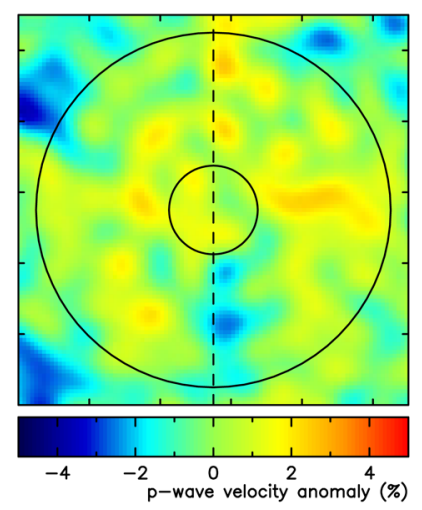

(c)

\section{Small scale velocity structure \\ (that is unknown)}

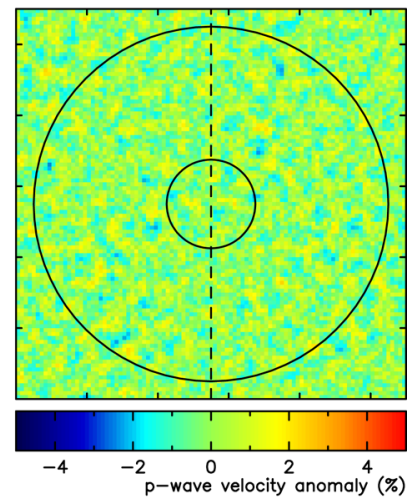

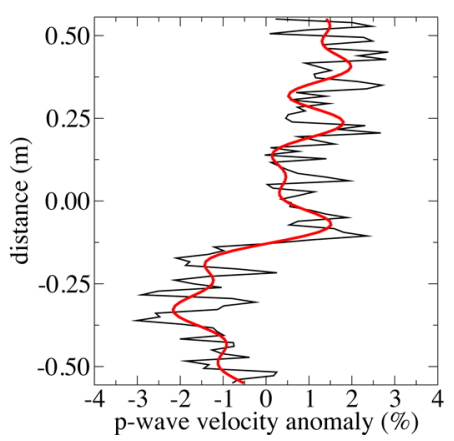

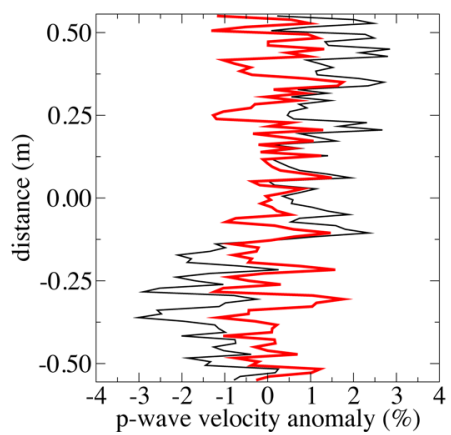

Figure 3. (a) The top panel represents a map of our test velocity model. The bottom panel is a velocity profile taken along the vertical dashed line in the top map. The velocity model is a computer generated self-affine random medium with Hurst exponent $H=-0.5$ which would correspond to a Brownian time-series in 1-D, also known as a random walk or a drunkard's walk (e.g. Klimeš 2002; Meschede \& Romanowicz 2015). (b) The top panel represents a map of the large-scale velocity structure that is assumed to be known a priori. It has been obtained by filtering the complete model in (a) in the Fourier domain, that is, by setting to zero the coefficients with wavenumber $k>2 \pi / \lambda_{c}$, where $\lambda_{c}$ is the spatial wavelength of the velocity anomalies. In our tests, we used $\lambda_{c}=12.5 \mathrm{~cm}$ that corresponds to the radius of the inner circle in which we want to image the velocity structure. The solid red line in the bottom panel is a velocity profile taken along the vertical dashed line in the top map and the solid black line shows the unfiltered velocity profile. (c) The top panel represents a map of the small scale velocity structure that is the unknown of our imaging problem. It has been obtained by filtering the complete model in (a) in the Fourier domain, that is, by setting to zero the coefficients with wavenumber $k \leq 2 \pi / \lambda_{c}$, where $\lambda_{c}$. The red solid line in the bottom panel is a velocity profile taken along the vertical dashed line in the top map and the black solid line shows the unfiltered velocity profile. In these experiments, all the sources and all the receivers are placed on the large outer circle and the velocity structure is imaged within the smaller inner circle.

uncorrelated variables. The standard deviation of the velocity anomaly error induced by the external structure (in Fig. 4d bottom panel) is $\sigma=0.39$. Though this error is about twice that of the model obtained without uncertainty about the external velocity structure, we think that the error in the final box model (in Fig. 4b) can be significantly reduced through regularization (that we did not introduce here). This is because the distribution of the error (in Fig. 4d) appears to be a high frequency uncorrelated noise which can be filtered out using a smoothing operator (e.g. Tarantola 1984).

This first example clearly shows that high resolution imaging (i.e. with resolution higher than that of the starting or reference model) of remote targets buried in an unknown medium is indeed feasible.

To assess how the burial depth affects the quality of the box image, we performed four series of inversions using a setup similar to the one shown in Fig. 3. In all series of inversions, the dimension of the imaging box, that is, the diameter of the inner circle was fixed to $0.1 \mathrm{~m}$, and, the diameter of the outer circle ranged from 0.1 to $0.9 \mathrm{~m}$. For each burial depth, that is, for each outer circle diameter, we generated ten realizations of the random medium. For each medium, we inverted the external traveltimes $\Delta \mathbf{d}^{\text {ext }}$ as discussed previously and we estimated amplitude of the error using

$\|\epsilon\|=\frac{\sqrt{\left(\mathbf{v}_{\Delta \mathbf{d}^{\text {ext }}}^{\text {box }}\right)^{T} \mathbf{v}_{\Delta \mathbf{d}^{\text {ext }}}^{\text {box }}}}{\sqrt{\left(\mathbf{v}^{\text {box }}\right)^{T} \mathbf{v}^{\text {box }}}}$

where $\mathbf{v}_{\Delta \mathbf{d}^{\text {ext }}}^{\text {box }}$ is the velocity error induced by the unknown external velocity, for example, as pictured in Fig. 4(d), and $\mathbf{v}^{\text {box }}$ is the target velocity, for example, as pictured in Fig. 4(a). Note that, as defined in eq. (20), $\|\epsilon\|$ measures the inverse of the signal to noise ratio in the local box-tomography image. The four series of inversions were performed the same way but using different sizes of data sets, that is, 
(a)

\section{true model}
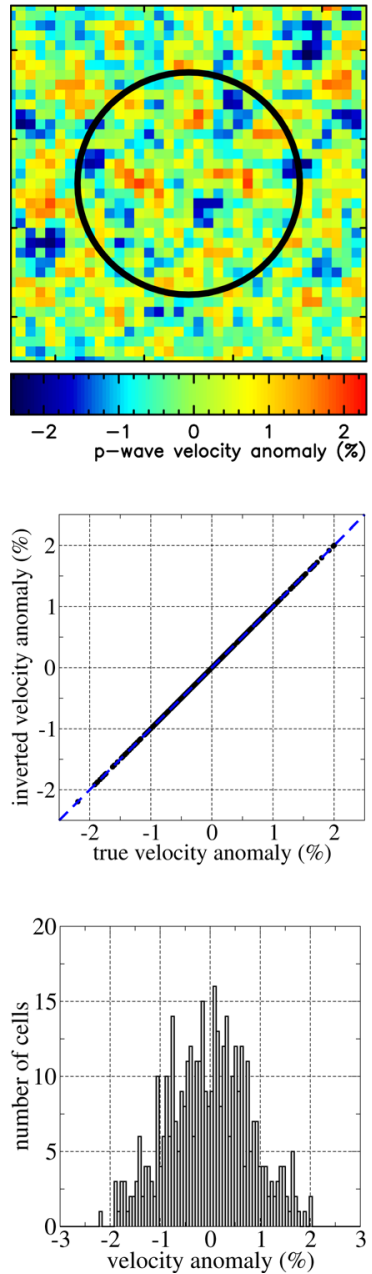

(b)

box model obtained with unknown external velocity (obtained by inverting $\left.\Delta \mathbf{d}^{\text {box }}+\Delta \mathbf{d}^{\text {ext }}\right)$
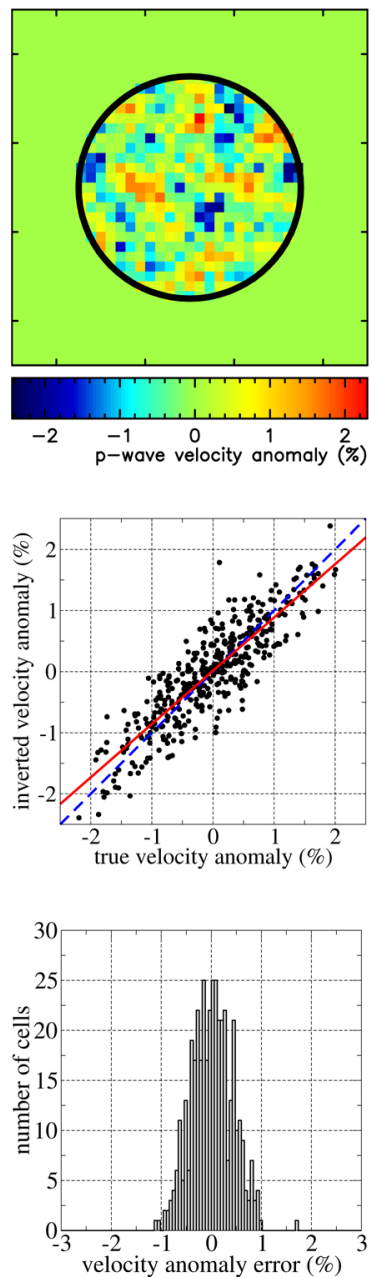

(c)

(d)

reference box model box error induced by obtained with known external velocity (obtained by inverting $\left.\Delta \mathbf{d}^{\text {box }}\right)$

the unknown external velocity (obtained by inverting $\left.\Delta \mathbf{d}^{\mathrm{ext}}\right)$
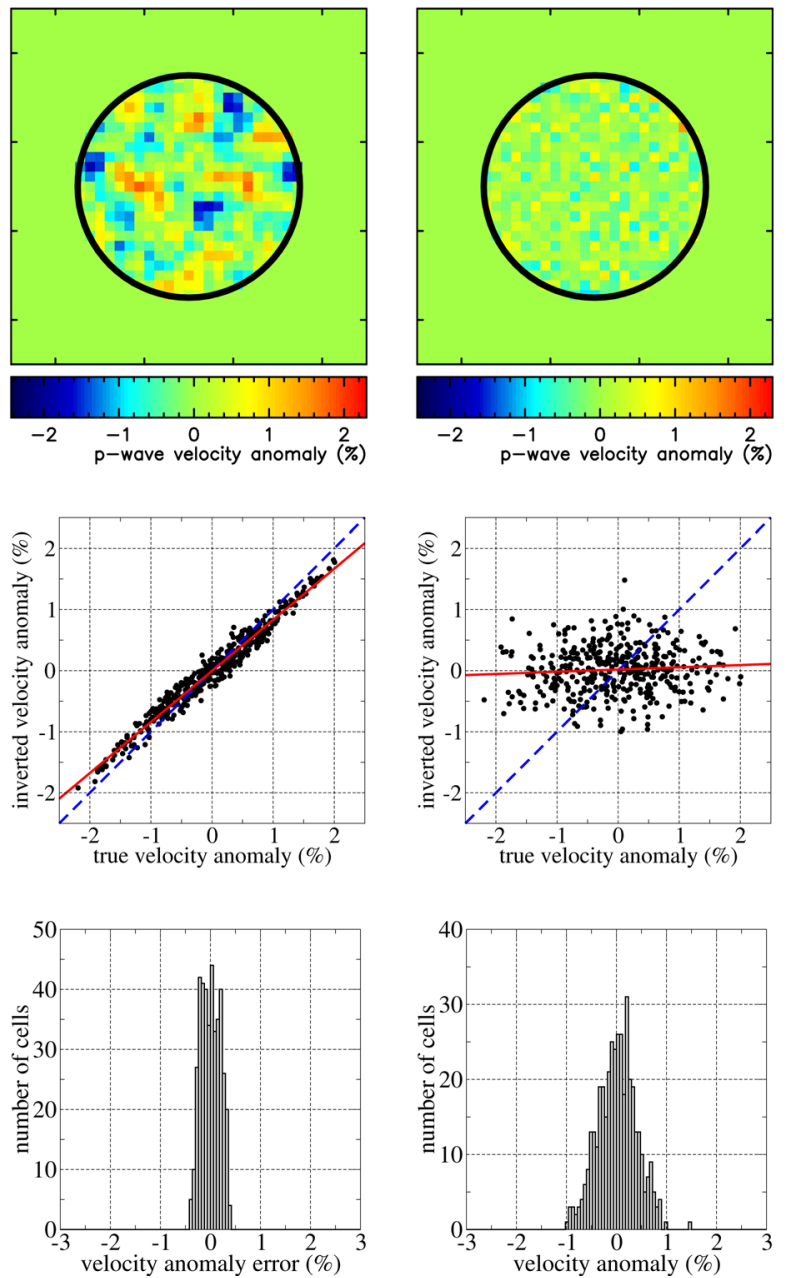

Figure 4. Results of the traveltime box-tomography inversions. The top panels present maps of the velocity obtained within the inner circle for the model in Fig. 3. These local maps are obtained by inverting distant data, that is, associated with sources and receivers placed on the outer circle in Fig. 3. The middle panels are scatter plots where the inverted velocities are plotted as a function of the true velocities within the imaging box delimited by the circle, the solid red lines correspond to linear regressions of the data, the dashed blue line is the identity line $y=x$. The middle panel in (a) shows the ideal scatter plot that would be obtained if the true velocity distribution were recovered by the inversion. The bottom panels in (a) and (d) are histograms showing the distribution of the velocity anomaly in the top maps, and, the bottom panels in (b) and (c) are histograms showing the distribution of the error in the velocity anomaly in the top maps (here the error is measured as the difference between the inverted velocity and the true velocity). (a) The target model, that is, the true velocity distribution. (b) Velocities obtained when inverting the total differential traveltimes, that is, using $\Delta \mathbf{d}^{\text {box }}+\Delta \mathbf{d}^{\text {ext }}$ in eq. (8). This is the complete box-tomography solution, it accurately reproduces the target model. (c) Velocities obtained when inverting the residual traveltimes induced by the unknown velocity structure inside the box, that is, using $\Delta \mathbf{d}^{\text {box }}$ and setting $\Delta \mathbf{d}^{\text {ext }}=0$ in eq. (8). This solution is also the solution of the local tomographic problem where the sources and the receivers are moved to the surface of the imaging box, it shows how well one can recover the target model with the data set considered. (d) Differences between the velocities in a) and the velocities in b), these velocities also correspond to those obtained when inverting the external residual traveltimes induced by the unknown velocity structure outside the box, that is, using $\Delta \mathbf{d}^{\text {ext }}$ and setting $\Delta \mathbf{d}^{\text {box }}=0$ in eq. (8). This shows the error in the velocities induced by the unknown velocity distribution outside the imaging box. The correlation coefficients corresponding the scatter plots in the middle panels in (a)-(d) are $r=1, r=0.87$, $r=0.98$ and $r=0.08$, respectively. The standard deviation of the distributions in the bottom panels in (a)-(d) are $\sigma=0.83, \sigma=0.41, \sigma=0.19$ and $\sigma=0.39$, respectively.

we varied the number of traveltimes/rays employed for the inversion from 1000 to 10000 . The results are presented in Fig. 5. Despite the noise in the observations, the error induced by the external structure appears to be roughly proportional to the square root of the burial depths in the depth range considered. The rate at which the error is increasing versus depth also depends on the size of the data set (i.e. the number of traveltimes used for the inversion). As the data set gets larger, the depth dependence gets smaller. For a given data set the maximum depth below which we can extract useful information about the target is reached when $\|\epsilon\|=1$. Our results suggest that 


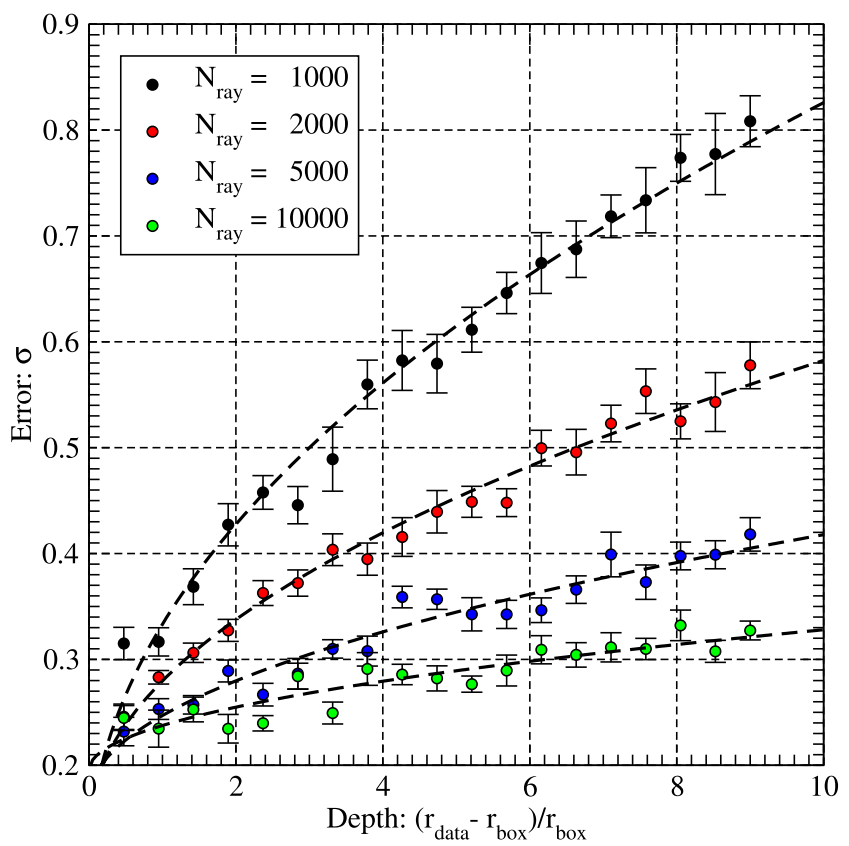

Figure 5. Evolution of the error $\|\epsilon\|$ as defined in eq. (20) induced by an unknown velocity structure outside the imaging box plotted as a function of the burial depth $\left(r_{\text {data }}-r_{\text {box }}\right) / r_{\text {box }}$. Each dot corresponds to an average value obtained from 10 measurements. The different colours correspond to different sizes of data set. The dashed lines show have been obtained by fitting the data using the template function $y=\mathrm{a} \sqrt{x}+\mathrm{b}$, where $\mathrm{a}$ and $\mathrm{b}$ are unknown coefficients.

using a dense enough data set sampling the box region, it should be possible to image targets at arbitrary depths. This behaviour is in good agreement with the results derived in the previous section.

\subsection{Localized imaging using waveform tomography}

In this section, we expand our discussion to the case of waveform tomography. While traveltime tomography uses first arrival times, waveform tomography, as its name suggests, uses waveforms or entire seismograms to image the medium. Here, we consider the acoustic problem where the waveforms $w(t)$ correspond to the pressure wavefield $p(\mathbf{x}, t)$ recorded at a set of receivers.

In order to compute the sensitivity functions in the case of waveform tomography, the Born approximation is generally used. Working with respect to a reference model with sound speed $c_{\text {ref }}(\mathbf{x})$, the Born perturbation can be computed for a perturbed medium with velocity $c(\mathbf{x})=c_{\text {ref }}(\mathbf{x})+\delta c(\mathbf{x})$ by taking the scattering integral

$\delta w(t)=\int_{V} K(\mathbf{x}, t) \delta c(\mathbf{x}) \mathrm{d} V$.

Eq. (21) in waveform tomography is the equivalent of eq. (1) in traveltime tomography. Note, however, that in waveform tomography the synthetic seismograms are usually computed by modelling wave propagation to obtain exact results and not using eq. (21) that gives approximate residual seismograms. Eq. (21) is only employed to linearize and solve the inverse problem. The sensitivity kernel or Fréchet derivative kernel $K(\mathbf{x}, t)$ tells us how, to first order, the synthetic data, that is, the waveform $w_{\text {ref }}(t)$, varies when one changes the model parameters, that is, when the velocity $c_{\text {ref }}(\mathbf{x})$ is perturbed at a given location $\mathbf{x}$. It plays the same role as the lengths $l_{i j}$ in eq. (1) that tell us how the traveltime $t_{i}$ along the $i$ th ray path varies when the slowness is modified within the $j$ th cell. The sensitivity kernel $K(\mathbf{x}, t)$ is formally defined as

$$
\begin{aligned}
K(\mathbf{x}, t) & =\frac{\partial p\left(\mathbf{x}_{r}, t\right)}{\partial c(\mathbf{x})} \\
& =\frac{1}{2 \pi} \int_{-\infty}^{\infty}-2 \omega^{2} G\left(\mathbf{x}_{s}, \mathbf{x}, \omega\right) G\left(\mathbf{x}_{r}, \mathbf{x}, \omega\right) \frac{S(\omega)}{c_{r e f}^{3}(\mathbf{x})} e^{i \omega t} \mathrm{~d} \omega
\end{aligned}
$$

where $\mathbf{x}_{s}$ and $\mathbf{x}_{r}$ are the positions of the source and the receiver, respectively, $S(\omega)$ is the spectrum of the source time function and the $G\left(\mathbf{x}_{i}, \mathbf{x}_{j}, \omega\right)$ are the Green's functions of the reference medium for source at $\mathbf{x}_{i}$ and receiver at $\mathbf{x}_{j}$. For a given source/receiver pair, the sensitivity kernel $K(\mathbf{x}, t)$ can be computed by modelling wave propagation numerically in the reference medium and running two simulations. A first simulation where an impulsive source is placed at the source position to obtain the wavefield $G\left(\mathbf{x}_{s}, \mathbf{x}, \omega\right)$ (e.g. as in Fig. 2c) and a second simulation where an impulsive source is placed at the receiver position to obtain the wavefield $G\left(\mathbf{x}_{r}, \mathbf{x}, \omega\right)$. By convolving the two wavefields we obtain $K(\mathbf{x}, t)$ that is often referred to as a banana doughnut kernel because of its shape illustrated in Fig. 2(e). When evaluated at the time of first arrival, the sensitivity kernel follows the ray path in Fig. 2(a), it is hollow when the source $S(\omega)$ has a finite frequency content, and, its thickness depends on the frequency content of the source (e.g. Dahlen et al. 2000). At later times, it becomes more spread as the waves travelled further away from the first arrival ray path. To setup the waveform tomography problem, one first needs to discretize and index the waveforms as a function of time. The data is treated as a set of $M$ scalar values $w_{i}$ ( $i \in 1, \ldots, M$ ) corresponding to the amplitude of a given waveform $w(t)=w\left(S^{i}, \mathbf{x}_{s}^{i}, \mathbf{x}_{r}^{i}, t^{i}\right)$ measured at position $\mathbf{x}_{r}^{i}$, for a source with spectrum $S^{i}$ and position $\mathbf{x}_{s}^{i}$ and at time $t^{i}$. Then, one discretizes the problem spatially, for example, by dividing the space into $N$ cells with constant velocity $c_{i}$. This way, eq. (21) becomes

$\delta w_{i}=\sum_{j} A_{i j} \delta c_{i}$,

where

$\mathbf{A}=A_{i j}=\int_{V_{j}} K_{i}(\mathbf{x}) \mathrm{d} V_{j}$,

is an $M \times N$ matrix, $V_{j}$ denotes the volume occupied by the $j$ th cell and $K_{i}(\mathbf{x})=K\left(c_{\text {ref }}, S^{i}, \mathbf{x}_{s}^{i}, \mathbf{x}_{r}^{i}, t^{i}\right)$ is the sensitivity kernel associated with the $i$ th data $w_{i}$. Each row in the matrix $\mathbf{A}$ contains a sensitivity kernel evaluated at time $t_{i}$ similar to the one in Fig. 2(e). Eq. (23) leads to a linear system similar to eq. (3) but where the vectors $\mathbf{d}$ and $\mathbf{d}_{n}$ contain the observed $w_{i}$ and the synthetic $w_{\text {ref } i}$ waveform amplitudes, respectively, and the vectors $\mathbf{m}_{n+1}$ and $\mathbf{m}_{n}$ contain the updated $c_{n+1 j}$ and current $c_{n j}$ velocities of the cells $j$ $\in 1, \ldots, M$, respectively. The waveform tomography problem can be solved iteratively like the traveltime tomography problem by evaluating eq. (4) recursively. Similarly, the solution to the box waveform tomography problem can be obtained using eq. (8).

To construct the matrix $\mathbf{A}_{\text {ref }}^{\text {box }}$ in eq. (8), we need the value of the sensitivity kernel within the imaging box only. This local sensitivity kernel can be computed efficiently as proposed by Masson \& Romanowicz (2017) using injection techniques combined with wavefield extrapolation. In this case, two local simulations similar to the one in Fig. 2(d) are needed to obtain the local sensitivity kernel, for example, in Fig. 2(f). Because wave propagation modelling can be completely confined within the imaging box once the Green's functions have been computed, the computational effort for imaging localized objects is much smaller than that needed to image the entire volume encompassing the sources and the receivers. 
This is what makes waveform box-tomography attractive for imaging localized targets. As in traveltime tomography, the local image is affected by the uncertainty on the velocity structure outside the imaging box.

To investigate how waveform box-tomography performs, we repeated the tests described in the previous section. The only difference with the inversions presented in the previous section being that the traveltime data have been replaced by waveform data. To construct the differential seismograms in the vectors $\Delta \mathbf{d}^{\text {box }}$ and $\Delta \mathbf{d}^{\text {ext }}$, we modelled wave propagation numerically (we solved the Helmholtz equation using a finite difference scheme). Note that we used an exact theory for the forward modelling together with the approximate sensitivity kernel in eq. (22) for solving the inverse problem. This approach is similar to that of Lekić \& Romanowicz (2011) who used spectral element simulations for computing synthetic seismograms and nonlinear asymptotic mode coupling theory (Li \& Romanowicz 1995) for the inversion. To construct the vector $\Delta \mathbf{d}^{\text {box }}$, we subtracted the wavefield obtained by modelling wave propagation in the medium with velocity distribution

$c_{\text {box }}(\mathbf{x})= \begin{cases}c_{\text {ref }}(\mathbf{x}) & \text { inside the imaging box } \\ c(\mathbf{x}) & \text { outside the imaging box }\end{cases}$

from the wavefield obtained by modelling wave propagation in the true model with velocity $c(\mathbf{x})$ (i.e. plotted in Fig. 3a). Similarly, to construct the vector $\Delta \mathbf{d}^{\text {ext }}$, we subtracted the wavefield obtained by modelling wave propagation in the medium with velocity distribution

$c_{\text {ext }}(\mathbf{x})= \begin{cases}c(\mathbf{x}) & \text { inside the imaging box } \\ c_{\text {ref }}(\mathbf{x}) & \text { outside the imaging box }\end{cases}$

from the wavefield obtained by modelling wave propagation in the true model with velocity $c(\mathbf{x})$. Just as for the traveltime inversions, we used a total of 20000 source/receiver pairs. We used complete waveforms so the data set here is also largely sensitive to the velocity structure outside the imaging box (as opposed to the traveltime case where we selected only the rays passing through the box). For each source/receiver pair, we recorded $1 \mathrm{~ms}$ waveforms between times $t_{1}=0.0 \mathrm{~s}$ and $t_{2}=0.001 \mathrm{~s}$ sampled at $133643 \mathrm{~Hz}\left(\Delta t=7.48 \times 10^{-6}\right.$ s). This correspond to $n=141$ samples $w_{i}$ per waveform.

The tomographic images obtained by inverting $\Delta \mathbf{d}^{\text {box }}$ and $\Delta \mathbf{d}^{\text {ext }}$ jointly and separately are presented in Fig. 6. Overall, the results are very similar to those obtained with traveltime tomography in Fig. 4.

The box image obtained when the external velocity structure is known (in Fig. 4c) almost perfectly reproduces the true velocity structure of the propagating medium. The estimated correlation coefficient is $r=0.99$ which is higher than the $r=0.98$ obtained with traveltime tomography. This is not surprising because waveforms carry more information than traveltimes. As in the case of traveltime tomography, the distribution in the scatter plot is slightly rotated clockwise since the absolute velocities are marginally underestimated by the inversion process.

The local image obtained by inverting $\Delta \mathbf{d}^{\text {ext }}$ alone (in Fig. $6 \mathrm{~d}$ ) has small velocity amplitude and the uncertainty in the external velocity model does not significantly map into the local image. The velocity map exhibits larger scale fluctuations when compared to the corresponding map obtained with traveltimes in Fig. 4(d). This is likely due to the fact that waveform tomography uses broadband data whereas traveltime tomography uses an infinite frequency approximation. Interestingly, the scatter plot distribution shows a slight anti-correlation between the inverted velocities and the true velocities. Though it is a little larger than that obtained with traveltimes, the measured correlation coefficient $r=-0.28$ is still relatively small.

The local image obtained by inverting $\Delta \mathbf{d}^{\text {box }}$ and $\Delta \mathbf{d}^{\text {ext }}$ jointly is of better quality than the one obtained with traveltime tomography and the visual match with the true velocity model is excellent. The correlation coefficient $r=0.91$ increased significantly compared to the $r=0.74$ obtained with traveltimes. This illustrates the power of waveform tomography compared to traveltime tomography, even in the case of random distribution of small scale heterogeneities.

Though we did not investigate the depth dependence in the case of waveform tomography (because of the heavy cost of the computations), we can derive a relation similar to that in eq. (16). In waveform tomography, we expect that the depth dependence in the error $\epsilon$ should be a lot less pronounced. This is because the scattered waves in the coda efficiently sample the entire space (i.e. even with a limited number of recordings), therefore, the standard error in the average slowness $\left\langle\Delta s^{\text {ext }}\right\rangle$ should be much smaller than in traveltime tomography.

So far, we showed that both traveltime box tomography and waveform box tomography can accurately image remote regions buried in a medium with unknown velocity distribution. However, we employed large enough data sets along with small velocity perturbations to avoid having to regularize the inverse problem and to iterate. Also, we employed an exact boundary condition for confining the wavefield within the imaging box in waveform box tomography, which may become too computationally intense when solving larger problems (see Masson \& Romanowicz 2017). In the next section, we consider more realistic situations, as encountered in the case of global seismology.

\section{FULL WAVEFORM BOX TOMOGRAPHY IN THE DEEP EARTH}

We now evaluate the interest of box tomography for imaging geological structure in the deep Earth. The framework is as follows:

On the one hand, the latest generation of global tomographic models provides us with 3-D images of the velocity structure inside the entire Earth but with a limited resolution. There are essentially two factors that limit the resolution of current global tomographic models (obtained by modelling wave propagation numerically), first, the uneven distribution of earthquakes and seismic stations at the surface of Earth, second, the enormous amount of calculation required to reach high enough frequencies for imaging smaller scale structures.

On the other hand, regional studies (at the continental scale or smaller) give partial images (say of the first few hundred kilometres below the Earth's surface) with higher resolution. This is because the computational effort is reduced as wave propagation is modelled in a smaller volume (i.e. much smaller than the total volume of the Earth).

As we have seen in the previous section, box tomography allows regional imaging at arbitrary location inside the Earth. Our objective here is to verify that we can achieve higher resolution than that of the current global models when imaging localized structures in the deep Earth.

In our tests, we consider a 2-D fluid planet, that has the same radius as the earth and realistic density and bulk modulus 1-D profiles as pictured in Fig. 7. We solve the tomographic problem using a standard full waveform inversion algorithm based on adjoint methods that is commonly used in regional studies (e.g. Bamberger 
(a)

\section{true model}
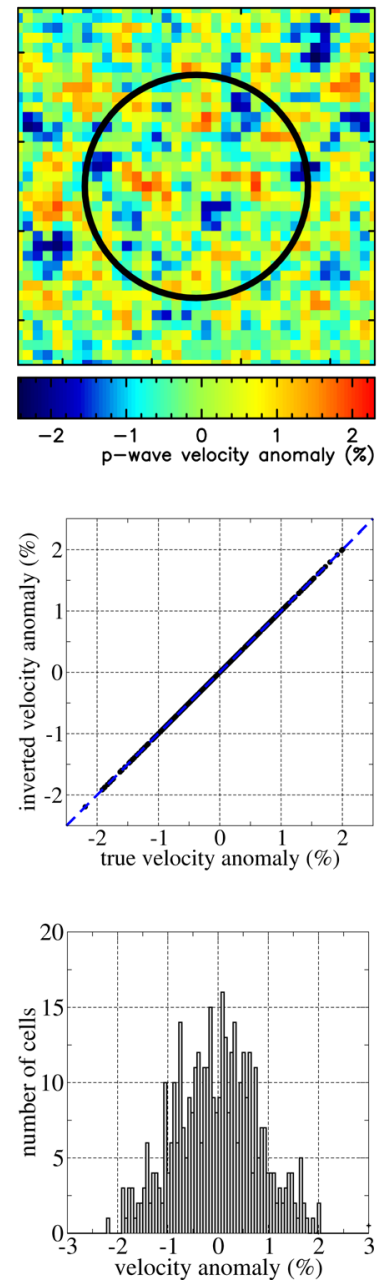

(b)

box model obtained with unknown external velocity

(obtained by inverting $\left.\Delta \mathbf{d}^{\text {box }}+\Delta \mathbf{d}^{\text {ext }}\right)$
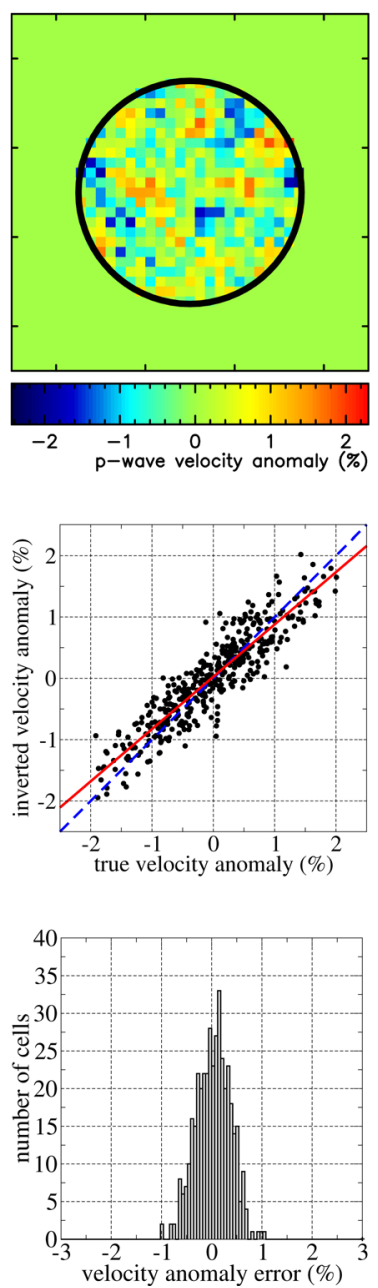

(c)

(d)

reference box model box error induced by obtained with known external velocity (obtained by inverting $\left.\Delta \mathbf{d}^{\text {box }}\right)$

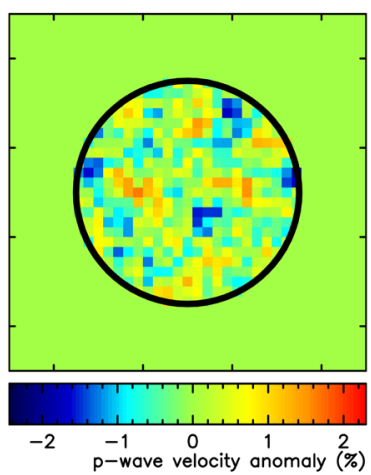

the unknown external velocity (obtained by inverting $\Delta d^{\text {ext }}$ )
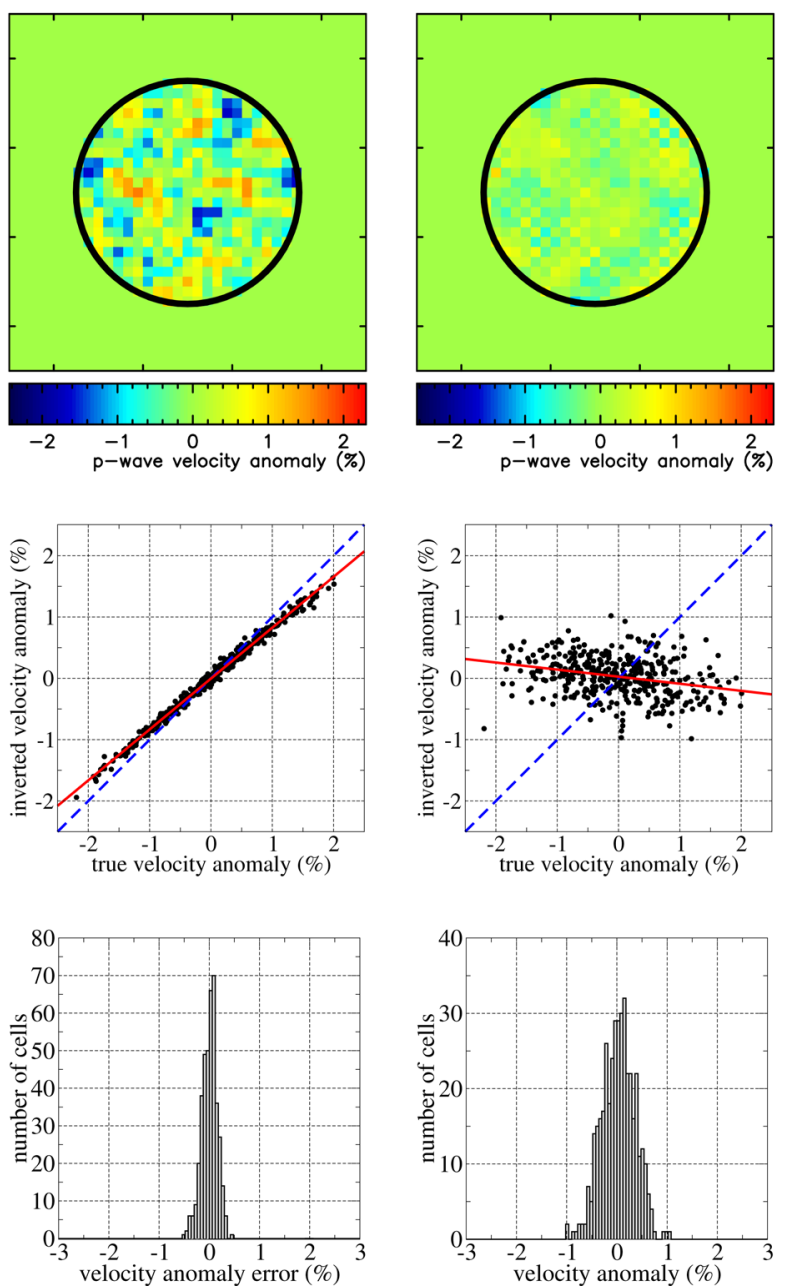

Figure 6. Results of the waveform box-tomography inversions. Same as Fig. 4 but for the case of waveform tomography.

et al. 1982; Tarantola 1984; Fichtner et al. 2006; Plessix 2006). We minimize the misfit functional:

$S=\left\|\mathbf{d}-\mathbf{d}_{\mathrm{ref}}\right\|^{2}$,

that is the $L^{2}$ norm of the difference between the observed seismograms $w(t)$ in $\mathbf{d}$ and the synthetic seismograms $w_{\text {ref }}(t)$ in $\mathbf{d}_{\text {ref }}$, using a steepest descent or conjugate-gradient scheme (Polak \& Ribiere 1969; Belina et al. 2009). At each iteration, the current model $\mathbf{m}_{\text {ref }}$ is updated using

$\mathbf{m}_{n+1}=\mathbf{m}_{n}+\eta_{n} \cdot \mathbf{c}_{n}$

where $\mathbf{c}_{\mathrm{n}}$ is the conjugate gradient and $\eta_{n}$ is the step length. In our tests, we calculated $\eta_{n}$ following Pica et al. (1990). The conjugate gradient is computed using (Polak \& Ribiere 1969)

$\mathbf{c}_{n}=\gamma_{n}+\frac{\gamma_{n}^{T}\left(\gamma_{n}-\gamma_{n-1}\right)}{\gamma_{n}^{T} \gamma_{n-1}} \mathbf{c}_{n-1}$ where $\gamma$ is the discretized version of the adjoint gradient

$\gamma_{n}(\mathbf{x})=k_{n}(\mathbf{x})^{2} \sum_{s=1}^{N_{s}} \int_{0}^{\infty} \frac{\partial p_{\mathrm{fwd}}}{\partial t}\left(\mathbf{x}_{s}, \mathbf{x}, t\right)_{n} \frac{\partial p_{\text {adj }}}{\partial t}\left(\mathbf{x}_{s}, \mathbf{x}, t\right)_{n} \mathrm{~d} t$

where $p_{\text {fwd }}$ is the forward propagated wavefield and $p_{\text {adj }}$ is the adjoint wavefield. The latter corresponds to the back-propagation in time of the difference between the observed $w(t)$ and the synthetic $w_{\text {ref }}(t)$ seismograms.

For global inversions, that is, where one searches for the velocity structure within the entire planet, the wavefields $p_{\mathrm{fwd}}$ and $p_{\text {adj }}$ need to be known everywhere and can be obtained through global wave propagation modelling. For performing a box inversion, one only needs to know the wavefields $p_{\text {fwd }}$ and $p_{\text {adj }}$ within the imaging box. The box problem can be solved by substituting $\mathbf{x}, \mathbf{c}, \gamma, p_{\text {fwd }}$ and $p_{\text {adj }}$ with $\mathbf{x}^{\text {box }}, \mathbf{c}^{\text {box }}, \gamma^{\text {box }}, p_{\text {fwd }}^{\text {box }}$ and $p_{\text {adj }}^{\text {box }}$ in eqs (28)-(30). During the box inversion, the wavefields $p_{\text {fwd }}^{\text {box }}$ and $p_{\text {adj }}^{\text {box }}$ can be computed exactly and efficiently using purely local simulations as proposed by Masson \& 


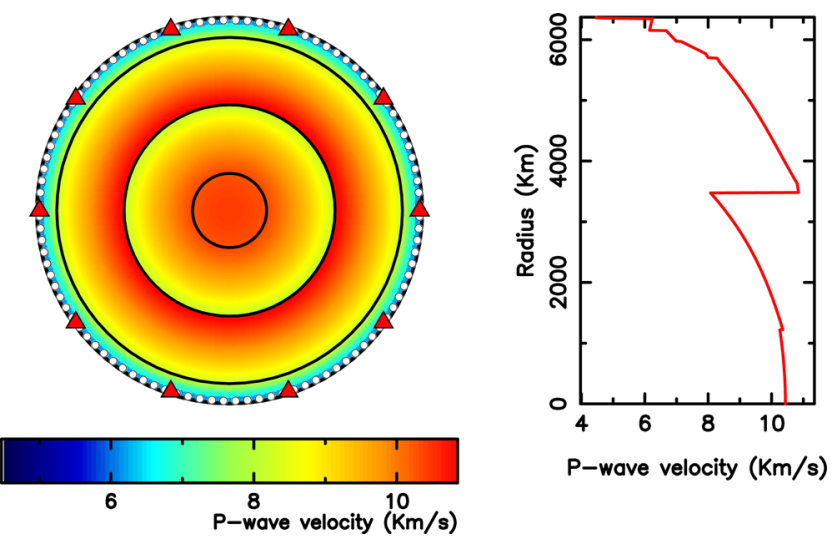

Figure 7. Acquisition setup and reference model employed for the tomographic inversions. We consider a 2-D fluid planet where only $P$-waves can propagate (i.e. an acoustic approximation). The planet has an axisymmetric velocity structure and has the same bulk-modulus profile, density profiles and radius as the Earth (as in the PREM model, Dziewonski et al. 1982). The seismic sources are represented by red triangles and the receivers are represented by white dots. Both the sources and the receivers are placed at a depth of $10 \mathrm{~km}$.

Romanowicz (2017). In this case, prior to the box inversion, the following global simulations need to be run to obtain the Green's function of the reference medium:

(i) $N_{s}$ global simulations to obtain the wavefields $p\left(\mathbf{x}_{s}, \mathbf{x}_{\sigma}, t\right)$ generated by a seismic sources with coordinates $\mathbf{x}_{s}\left(s=1, \ldots, N_{s}\right)$ and evaluated at the grid points with coordinates $\mathbf{x}_{\sigma}(\sigma=1, \ldots$, $N_{\sigma}$ ) located at the surface $\partial V_{\text {box }}$ of the imaging box.

(ii) $N_{r}$ global simulations $\left(3 \times N_{r}\right.$ in the elastic case) to obtain the Green's functions $G\left(\mathbf{x}_{r}, \mathbf{x}_{\sigma}\right)$ between each receiver with coordinates $\mathbf{x}_{r}$ and the grid points with coordinates $\mathbf{x}_{\sigma}\left(\sigma=1, \ldots, N_{\sigma}\right)$ located at the surface $\partial V_{\text {box }}$ of the imaging box.

(iii) $N_{\sigma}$ global simulations ( $3 \times N_{\sigma}$ in the elastic case) to obtain the Green's functions $G\left(\mathbf{x}_{\sigma_{1}}, \mathbf{x}_{\sigma_{2}}\right)$ between all possible pairs of points $\left(\mathbf{x}_{\sigma_{1}}, \mathbf{x}_{\sigma_{2}}\right)$, where $1<\sigma_{1}<N_{\sigma}$ and $1<\sigma_{2}<N_{\sigma}$, lying on the surface $\partial V_{\text {box }}$ of the imaging box.

When following Masson \& Romanowicz (2017), the points with coordinates $\mathbf{x}_{\sigma}$ form a layer a few grid points thick, which follows the surface $\partial V_{\text {box }}$ of the imaging box. In this case the total number of points $N_{\sigma}$ is prescribed by the numerical solver employed for modelling wave propagation (i.e. these points belong to the coallocation points of the numerical mesh). In general, $N_{\sigma}$ is imposed by the Nyquist criterion, that is, the wavefield at the surface of the imaging box must be sampled using at least two points per spatial wavelength (see Masson et al. 2014). In most situations, we have $N_{\sigma} \gg N_{s}+N_{r}$ and most of the computational effort (prior the box tomography inversion) is spent running global simulations to obtain the Green's functions $G\left(\mathbf{x}_{\sigma_{1}}, \mathbf{x}_{\sigma_{2}}\right)$. When $N_{\sigma}$ gets too high, the computational cost of these global simulations may become large and limit the efficiency of box tomography. Fortunately, using an approximate solution to the scattering problem, where higher order scattering effects are neglected (see Masson \& Romanowicz 2017), the total number of global simulations needed prior to running the local inversion can be reduced to $N_{s}+N_{r}$. Practically, this is achieved by replacing the exact boundary condition that confines the wavefield within the imaging box by an absorbing boundary. In this case, waves generated by velocity perturbations inside the imaging box, that escape the local modelling domain, interact with the structure outside the modelling domain, and get scattered back inside the modelling domain, will not be present or visible in the computed waveforms. All the examples we introduce in this section have been obtained by neglecting the higher order multiple scattering effects, in order to demonstrate that accurate images can be obtained efficiently without implementing an exact boundary condition, as suggested by Masson \& Romanowicz (2017).

\subsection{Localized imaging in a planet with known velocity structure}

We first verify that our numerical scheme performs well when the velocity structure in known within the planet (to the exception of the target structure). To do so, we constructed the target model pictured in the bottom panel of Fig. 8 by adding positive velocity anomalies to the reference model pictured in Fig. 7 at a depth corresponding to the base of the Earth mantle.

We generated a synthetic data set by modelling wave propagation in the target model using a finite difference scheme. We ran one simulation for each one of the ten seismic sources marked by the red triangles in Fig. 7. In each simulation, we recorded the wavefield at a hundred receivers located at the surface of the planet (marked by white circles in Fig. 7). Thus, our data set consists of 1000 seismograms with duration $1600 \mathrm{~s}$. These seismograms were filtered before the inversion using a low pass filter with a cut-off frequency corresponding to a period of $20 \mathrm{~s}$.

For testing box tomography against global tomography, we ran two tomographic inversions, a global inversion where we inverted the velocity in the entire planet and a local inversion where we inverted the velocity inside the rectangular box delimited by the dashed line in Fig. 8. We used the same setup in both inversions. We performed 15 iterations using the conjugate gradient scheme in eq. (28). To regularize the inverse problem, at each iteration step, we smoothed out the gradient $\gamma(\mathbf{x})$ using a moving average filter, we used a cosine window with spatial extents $\lambda_{n} \times \lambda_{n}$, where $n$ is the iteration index, for the filtering. We started with $\lambda_{1}=1500 \mathrm{Km}$ and we progressively released the gradient smoothing constraint using $\lambda_{n}=\frac{4}{5} \times \lambda_{n-1}$. Thus the maximum resolution of the final models after 15 iterations is about $\lambda_{15} \approx 50 \mathrm{Km}$. In order to give similar importance to the different waves (e.g. surface waves, body waves, scattered waves, etc.), we weighted the amplitudes of the observed and synthetic seismograms (i.e. when evaluating the misfit function and computing the adjoint sources (e.g. Li \& Romanowicz 1996).

The tomographic images obtained with the global and the box inversions after 5 and 15 iterations are compared in Fig. 8. Both inversions converge to an accurate solution and recover the target model very well, though, the image obtained with box tomography better matches the target model visually. The evolution of the misfit function as a function of the number of iterations is pictured in Fig. 9 and we observe no significant difference between the convergence rates of the two inversions. To quantify the resemblance between the tomographic images and the target model, we measured the correlation coefficients $r=0.936$ for the global inversion and $r=0.982$ for the box inversion, which confirms that the local model is more accurate than the global model (within the imaging box). On the one hand, this is not surprising because we accounted for more a priori information in the box inversion, since the velocity structure is known outside the imaging box in the local inversion and not in the global one. On the other hand, however, it is remarkable that the box inversion performs better than the global one because an approximate theory has been used for computing the 
global tomography
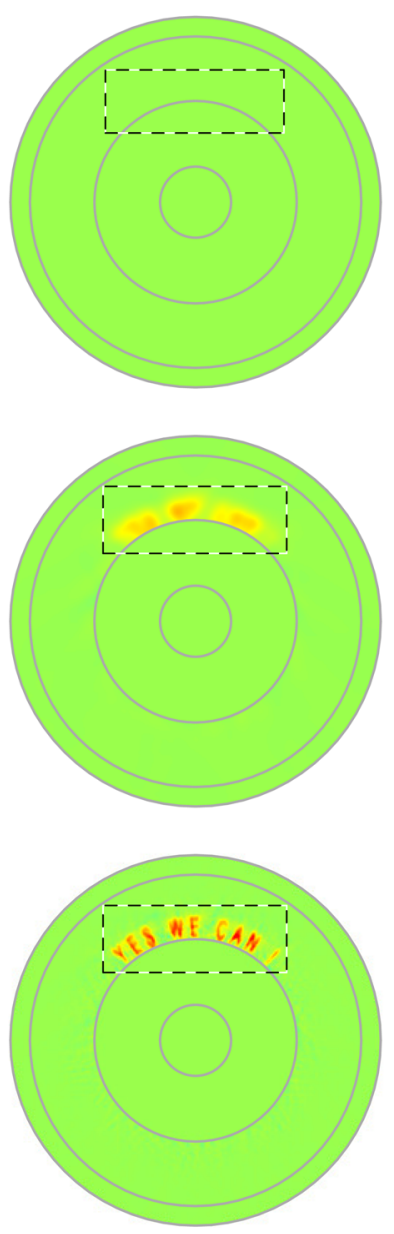

\section{box tomography}

iteration 0 starting/reference model

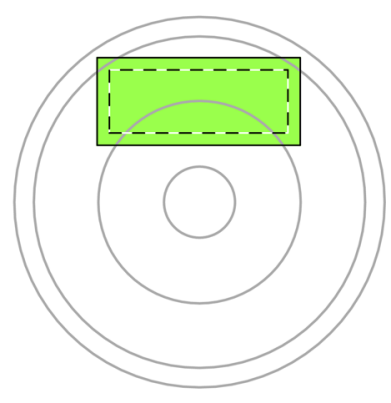

iteration 5

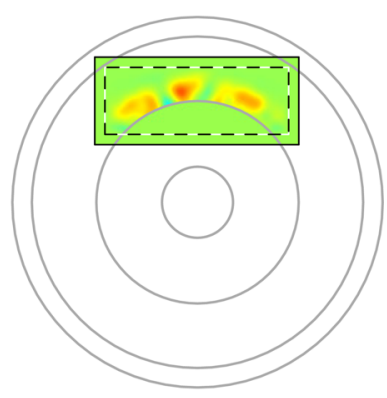

iteration 15

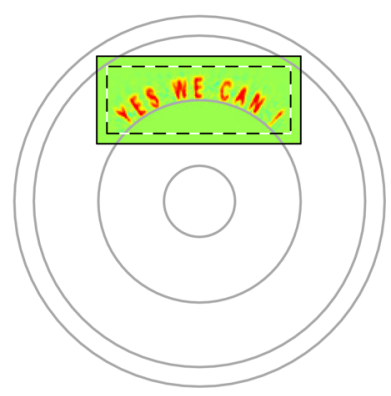

target model

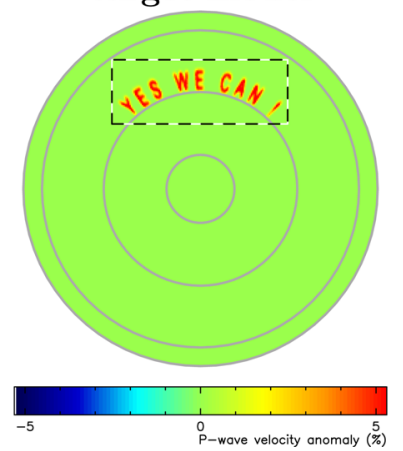

Figure 8. Comparison between a global tomographic inversion and a local tomographic inversion (within the box delimited by the dashed line) in the situation where the velocity structure outside the inversion box is fully known a priori. The reference or starting model employed for the inversion is pictured in Fig. 7. To build our data set, for ten seismic sources, we recorded the wavefield at a hundred seismic stations. The seismograms with duration $1600 \mathrm{~s}$ were filtered before the inversion using a low pass filter with cut-off frequency corresponding to a period of $20 \mathrm{~s}$. For the inversion, we used an adjoint conjugate gradient scheme. The maximum resolution allowed by the spatial smoothing of the adjoint gradient varies from $1500 \mathrm{~km}$ at iteration 1 to $50 \mathrm{~km}$ at iteration 15 . After 15 iterations, both the global and the box-tomography models converge and perfectly match the target model pictured in the bottom panel

synthetic seismograms (i.e. we neglected the higher order multiple scattering effects by putting an absorbing boundary at the surface of the imaging box). In Fig. 10, we compare the scatter plots obtained with the two inversions. Interestingly, the distribution associated with the global model has greater spread, also the linear regression (solid red line) shows that the inverted velocities are biased in the sense that their magnitude is underestimated. In the box inversion, the velocity distribution spread is significantly smaller and the bias is almost non-existent.

In summary, when the velocity structure in known outside the imaging box, box tomography outperforms global tomography as more information is available a priori. In the next example we 


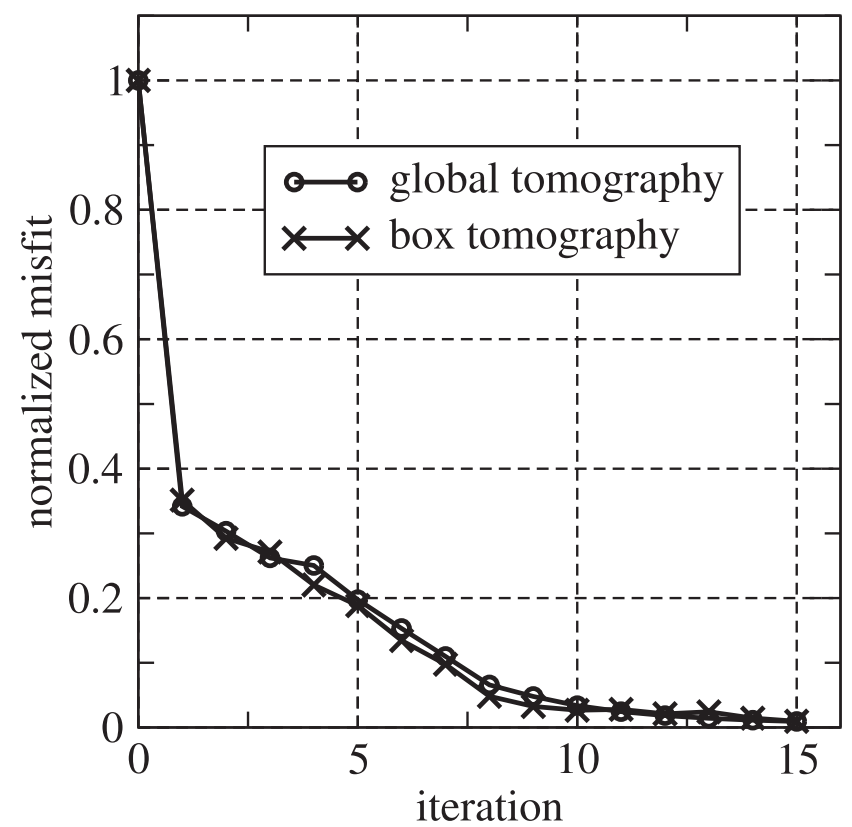

Figure 9. Evolution of the misfit function in eq. (27) as a function of the number of iterations in the situation where the velocity structure is known outside the imaging box. The circles correspond to the global inversion pictured in the left panels of Fig. 8. The crosses correspond to the local inversion pictured in the right panels of Fig. 8.

consider the situation where no a priori information is available about the velocity structure outside the imaging box.

\subsection{Localized imaging in a planet with unknown velocity structure}

In the previous example, we showed that box tomography performs well (i.e. even better than global tomography) when the velocity structure is known around the target region. We now push things further by introducing unknown velocity perturbations outside the imaging box. We constructed the target model in Fig. 11 that is a non-stationary random model with a locally Gaussian spatial correlation function (see Meschede \& Romanowicz 2015). The velocity distribution is Gaussian at the surface of the earth and transforms to a bimodal distribution (i.e. where the local velocity is the average velocity plus or minus 4 per cent) at depth to mimic what is observed in current global tomographic models. The objective here is to recover the velocity structure of the target model within the imaging box (delimited by the solid dashed line) using only a priori information contained in the reference model in Fig. 7.

We generated a synthetic data set by modelling wave propagation in the target model as in the previous example. For 20 seismic sources, we recorded the wavefield at 50 receivers with a geometry similar to what is shown in Fig. 7. We filtered the seismograms with duration $1600 \mathrm{~s}$ using a low pass filter with a cut-off frequency corresponding to a period of $25 \mathrm{~s}$. Here too, we performed a local and a global inversion using similar setups. For the regularization, we started with $\lambda_{1}=1000 \mathrm{~km}$ and we progressively released the gradient smoothing constraint using $\lambda_{n}=0.95 \times \lambda_{n-1}$. The maximum resolution of the final models after 15 iteration is about $\lambda_{15} \approx$ $400 \mathrm{~km}$.

The tomographic images obtained with the global and the local inversions after 3 and 15 iterations are compared in Fig. 11. As one (a) global tomography

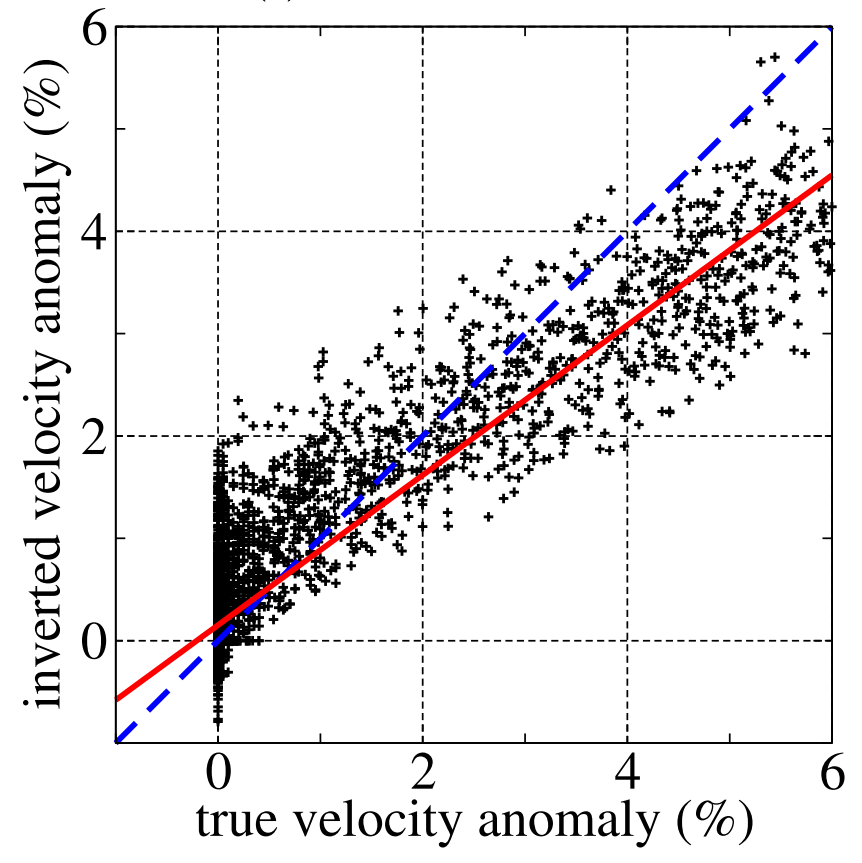

(b) box tomography

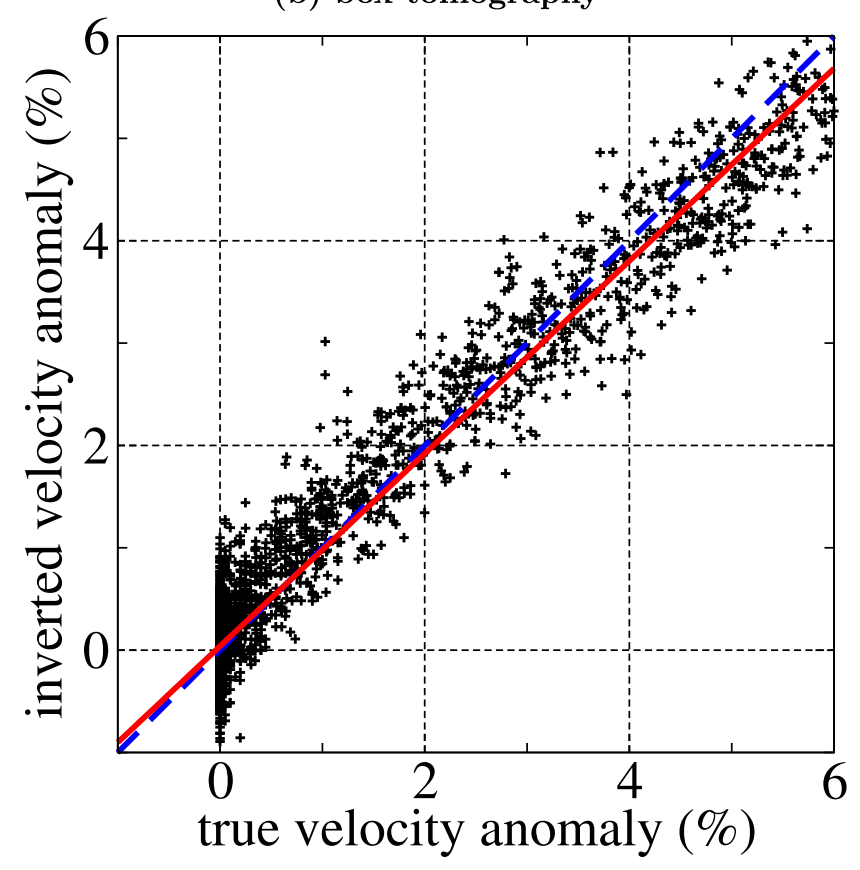

Figure 10. Scatter plots showing the correlation between the inverted velocities and the true velocities in the situation where the velocity structure is known outside the imaging box. The black dots show the inverted velocities plotted as a function of the true velocities of the medium (target model) inside the imaging box delimited by the dashed line in Fig. 8. The solid red line corresponds to the linear regression of the data. In the ideal situation where the true velocities are recovered by the inversion, all the dots lie on the dashed blue line of identity $y=x$. (a) Corresponds to the velocity model obtained with the global inversion pictured in the left panels of Fig. 8. (b) Corresponds to the velocity model obtained with the local inversion pictured in the right panels of Fig. 8. 
global tomography
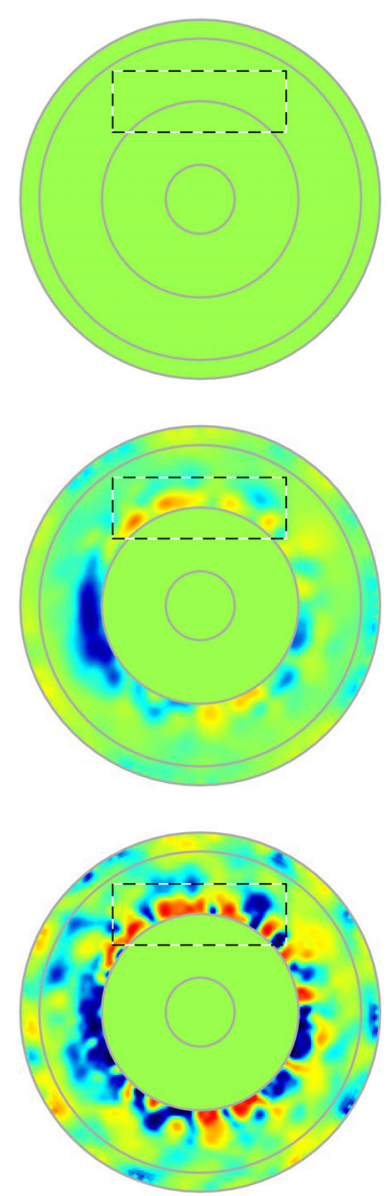

box tomography

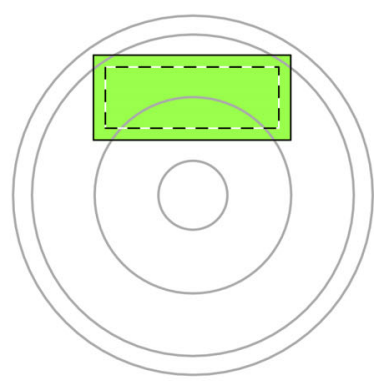

iteration 3

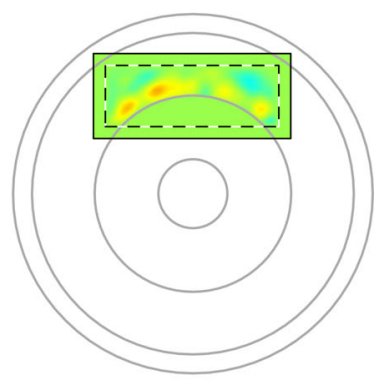

iteration 15

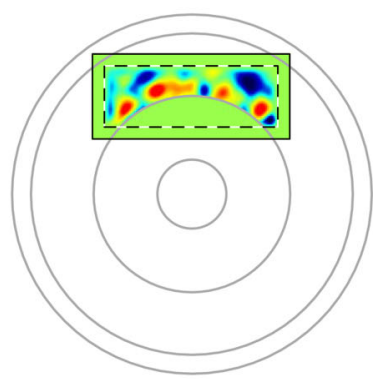

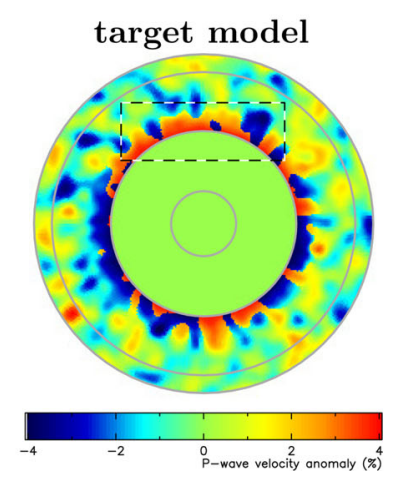

Figure 11. Comparison between a global tomographic inversion and a local tomographic inversion (within the box delimited by the dashed line) in the situation where the velocity structure outside the inversion box is not known a priori. The reference or starting model employed for the inversion is pictured in Fig. 7. To build our data set, for ten seismic sources, we recorded the wavefield at fifty seismic stations, the seismograms with duration $1600 \mathrm{~s}$ were filtered before the inversion using a low pass filter with cut-off frequency corresponding to a period of $33 \mathrm{~s}$. For the inversion, we used an adjoint conjugate gradient scheme. The maximum resolution allowed by the spatial smoothing of the adjoint gradient varies from $1000 \mathrm{~km}$ at iteration 1 to $300 \mathrm{~km}$ at iteration 15 . After 15 iterations, both the global and the box-tomography models give meaningful images that truly represent the target model pictured in the bottom panel. Though the box-tomography model is not as good as the global model, all the anomalies present in the true model are visible, well placed and have the correct sign. This is remarkable given that we used no information about the velocity structure outside the imaging box in the inversion.

might expect, the global inversion converges to an accurate model that well reproduces the shapes and, to a lesser extent, the amplitudes of the velocity anomalies present in the true model. What is remarkable, however, is that the local inversion also gives an excellent image of the structure within the imaging box. Though their shape slightly differ, all the anomalies present in the true model are visible, well placed and have the correct sign. Within the imaging box, we measured the correlation coefficients $r=0.917$ for the global inversion and $r=0.725$ for the local inversion. Not surprisingly, the global inversion performs significantly better because it corrects for the velocity anomalies located outside the imaging box as the model converges. Still, the correlation coefficient measured for the box model is relatively high which proves that useful information about the target structure has been extracted from the data 
(a) global tomography

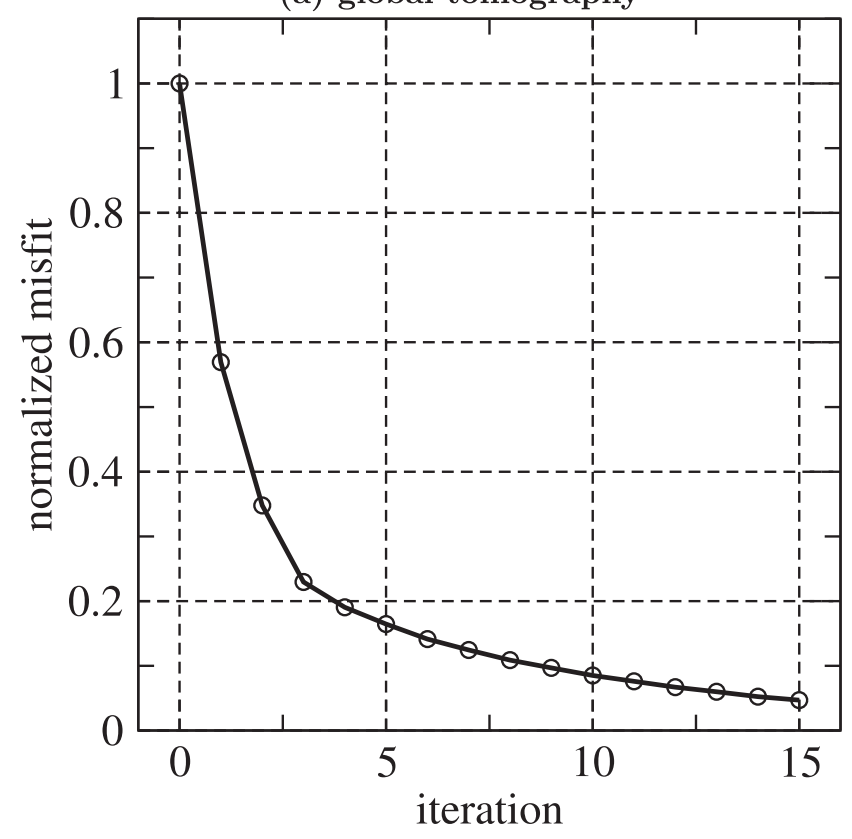

(b) box tomography

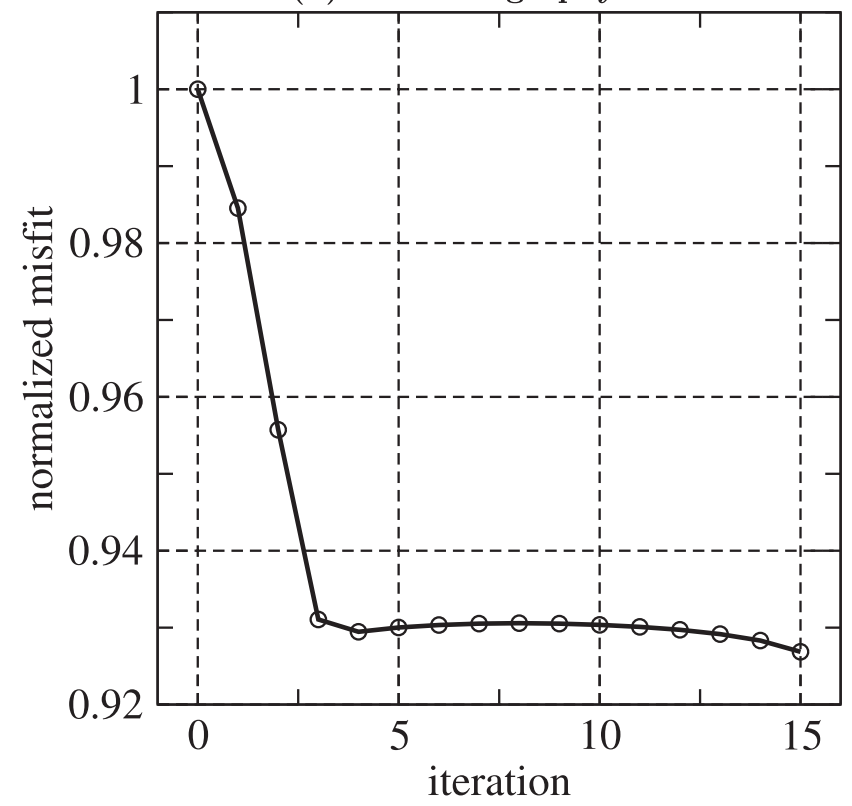

Figure 12. Evolution of the misfit function in eq. (27) as a function of the number of iterations in the situation where the velocity structure is not known outside the imaging box. (a) Corresponds to the global inversion pictured in the left panels of Fig. 11. (b) Corresponds to the local inversion pictured in the right panels of Fig. 11.

during the inversion. Looking at the evolution of the misfit function in Fig. 12, we see that this happens even though the value of misfit function only decreases by 7 per cent in the local inversion as opposed to about 95 per cent in the global inversion. This is because the data are principally sensitive to the structure outside the target region. Interestingly, the local inversion converges faster and reaches a plateau after a few iterations. In Fig. 13, the scatter plots show that the distribution associated with the local model has greater spread than that of the global model. Here, both distributions are at an angle (i.e. the linear regression line in red is (a) global tomography

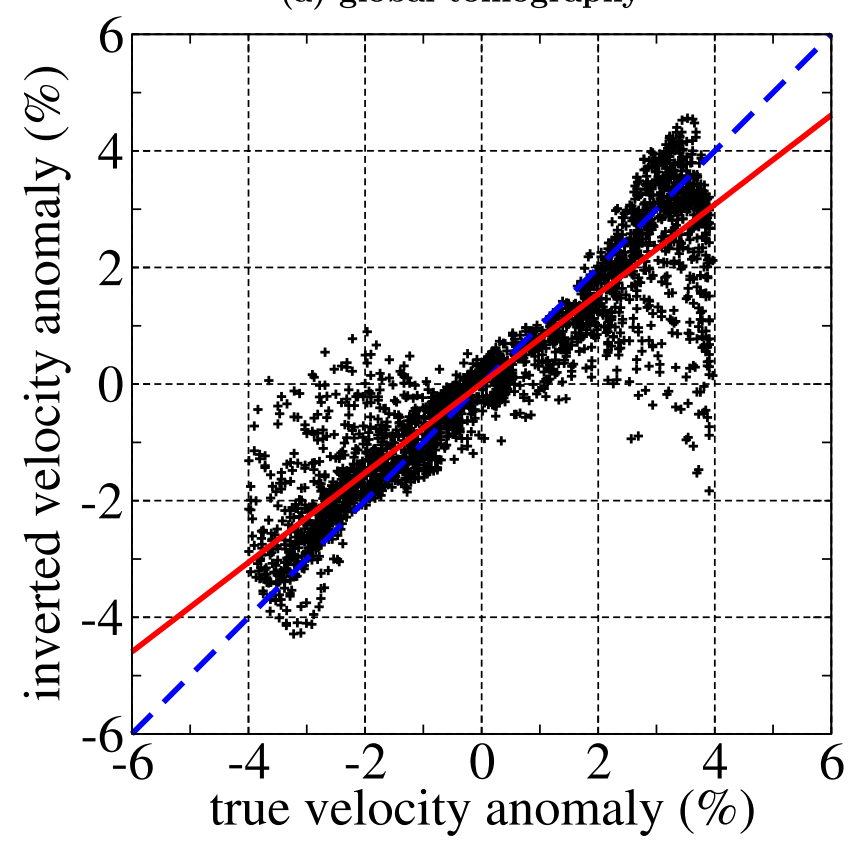

(b) box tomography

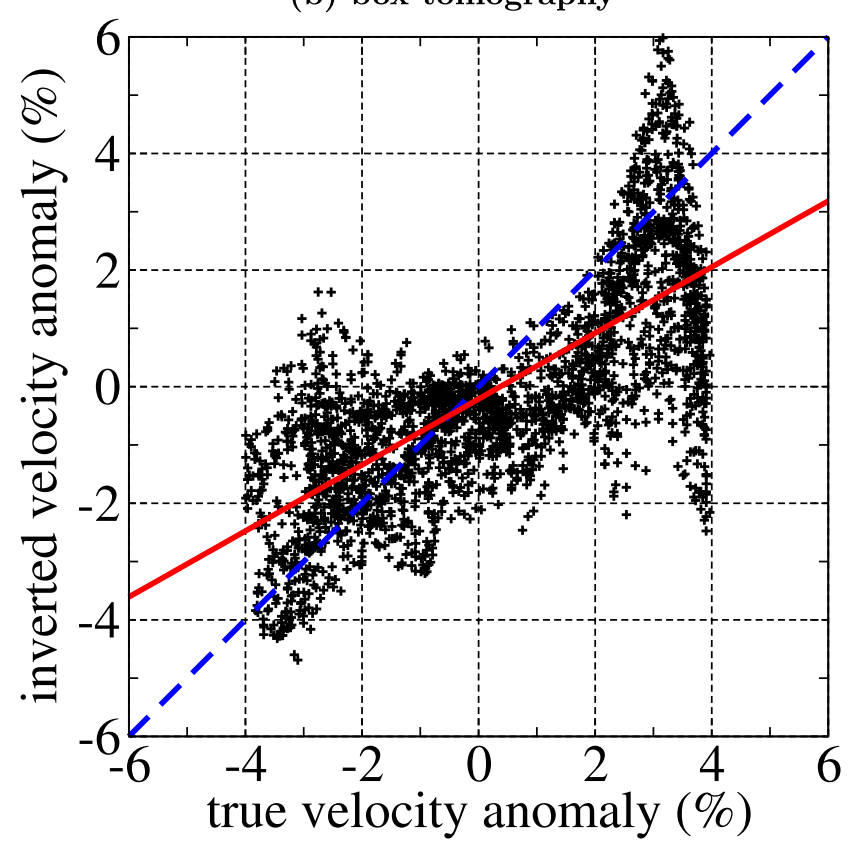

Figure 13. Scatter plots showing the correlation between the inverted velocities and the true velocities in the situation where the velocity structure is not known outside the imaging box. The black dots show the inverted velocities plotted as a function of the true velocities in the medium (target model) inside the imaging box delimited by the dashed line in Fig. 8. The solid red line correspond to the linear regression of the data. In the ideal situation where the true velocities are recovered by the inversion, all the dots lie onto the dashed blue line of identity $y=x$. (a) Corresponds to the velocities obtained with the global inversion pictured in the left panels of Fig. 11. (b) Corresponds to the velocities obtained with the local inversion pictured in the right panels of Fig. 11. 
rotated clockwise with respect to the reference dashed blue identity line), the angle being more pronounced in the box tomography case. Therefore, the magnitudes of the velocities are slightly more underestimated in the box model than in the global model. Note the negative vertical offset of the distribution associated with box tomography (i.e. the solid red line do not pass through the origin which is the case in all the other examples presented in this paper). This bias must be due to the fact that the velocity anomaly distribution (i.e. the difference between the true velocity and the velocity of the reference model) does not have zero mean outside the imaging box. Though we did not investigate this point, this vertical offset could be used to estimate the quality of the box and reference models.

The present example clearly demonstrates that box tomography allows the recovery of the velocity structure within a target region at depth, when almost no information about the velocity structure surrounding the imaging box is available a priori. This is in a way a worst case scenario. In the next example, we consider the more realistic situation where the a priori velocity structure outside the imaging box is first obtained using a global inversion with low spatial resolution.

\subsection{Localized imaging in a planet with partially known velocity structure}

As we have just seen, in the situation where we have no or little information about the 3-D velocity distribution of the medium surrounding the target region, it is still possible to obtain meaningful local tomographic images, however, these images might be subject to some bias. We now consider a realistic situation where the reference model employed for the box tomography inversion is the result of a previous global inversion with coarse resolution.

We constructed a 2-D reference model by running a global inversion exactly as described in the previous section to the exception that the inverted seismograms were filtered using a low pass filter with a cut-off frequency corresponding to a period of $300 \mathrm{~s}$. We intentionally employed periods longer than that used for constructing the current global tomographic models (say $60 \mathrm{~s}$ ) to place ourselves in a challenging situation. The reference model obtained is presented in the top left panel of Fig. 14 and is smooth because of the low frequency content of the data (i.e. not because of the regularization of the inversion, i.e. the gradient smoothing).

Using the smooth reference model, we again performed the two inversions described in the previous section using the exact same procedure. The images obtained after 3 and 15 iterations are presented in Fig. 14. When comparing these models to those obtained in the previous section (i.e. pictured in Fig. 12 and obtained starting with the 1-D reference model in Fig. 7), we observe significant improvement. In both models, the shapes and the amplitudes of the velocity anomalies better match those of the target model, though the local model appears a bit noisier. The correlation coefficients of the global and local models are $r=0.938$ and $r=0.864$, respectively. With respect to the smooth reference model with correlation coefficient $r=0.771$, the global and local models improved by 21 per cent and 12 per cent, respectively. With respect to the previous models (i.e. pictured in Fig. 12 and obtained starting with the 1-D reference model in Fig. 7), the global and local models improved by 2 per cent and 19 per cent, respectively. As in the previous example, the decrease of the misfit function pictured in Fig. 15 is small in the local case, because the data are mostly sensitive to the structure outside the target region. In the box tomography inversion, the misfit func- tion reaches a plateau after a few iterations and slowly decreases after that. Interestingly the best local or box model, that is, the one with the highest correlation coefficient $r=0.866$, is obtained after three iterations precisely where the misfit function reaches the plateau level (this model is visible in Fig. 14, i.e. iteration 3 to the right). The scatter plots in Fig. 16 show better correlation with the target model compared to those in Fig. 13. Though both distributions still underestimate the true velocities, the bias, as measured by the angle between the solid red line and the dashed blue identity line, is less important and is about the same in the box and global models. Note that the vertical offset of the box tomography distribution in Fig. 16(b) is significantly smaller than in Fig. 13(b). This confirms that, when using a homogenized 3-D model obtained from a previous inversion as the reference model, the unknown velocity structure outside the imaging box effectively averages to zero and does not significantly bias the local image, as we conjectured in the first section of this paper.

With this last example we showed that it is possible to efficiently refine global tomographic models locally using box tomography. Both the spatial resolution and the amplitude resolution of the box models are significantly higher than that of the starting or reference global model. Such improvements are critical for the geodynamic interpretation of the tomographic images in combination with mineral physics constraints.

\section{CONCLUSIONS}

We introduced and evaluated the box-tomography concept which permits to image localized geological structures buried in an unknown medium. We implemented three different flavours of boxtomography based on ray theory, scattering integrals and adjoint methods. Whatever the chosen approach, we showed that boxtomography can produce high resolution images of local structures in the difficult situation where no or little prior information about the velocity distribution within the medium is available. Such images can be obtained very efficiently as the numerical modelling, that is the ray tracing when working with traveltimes and numerical wave propagation modelling when working with seismograms, is completely confined within the small box volume to be imaged (except for a limited number of initial global simulations). Through simple analysis and numerical examples, we showed that, for a given data set, the quality of the local images obtained using box-tomography slowly degrades with the depth of the target. However, this effect can be annihilated using a dense enough data set, and accurate images can in theory be obtained at arbitrary depth.

Box-tomography is a method of interest whenever one need to refine an existing tomographic model locally, for example, to zoom around and infer the structure of reservoir rocks in exploration geophysics. In global seismology, box-tomography can be used to focus on key structures in the Earth's deep mantle. It appears well adapted for this task because the spectrum of the velocity anomalies and its power changes between the uppermost mantle, the midmantle and the $\mathrm{D}^{\prime \prime}$ region, with much stronger heterogeneity in $\mathrm{D}^{\prime \prime}$ than in the rest of the lower mantle. For example, box-tomography will allow us to extend the resolution limit in targeted regions of geophysical interest such as, for example, the roots of mantle plumes (e.g. French \& Romanowicz 2015). Even though we will not achieve the ultimate resolution, it will help define the outlines of structures of interest that can further be investigated using refined forward modelling methods, for which the model space sampling is currently limited due to the heavy nature of the numerical wavefield 
global tomography
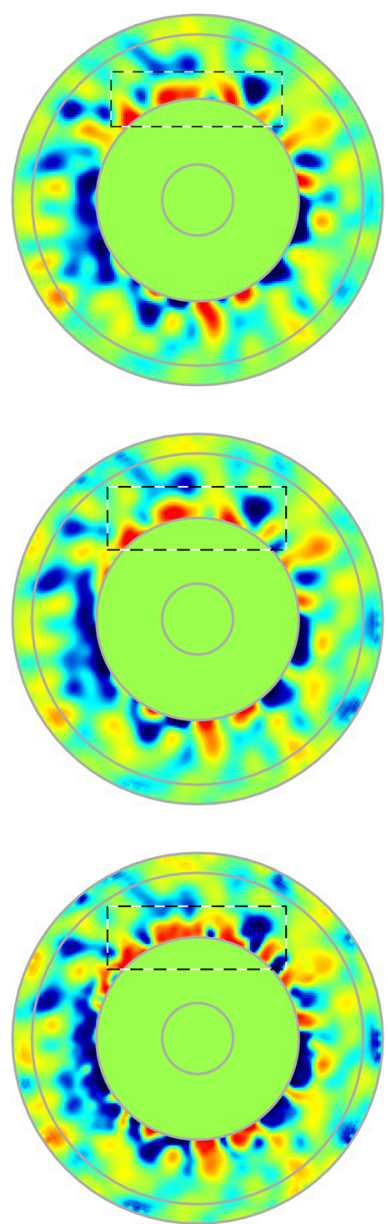

box tomography

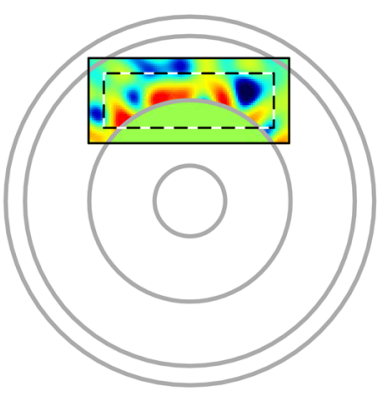

iteration 3

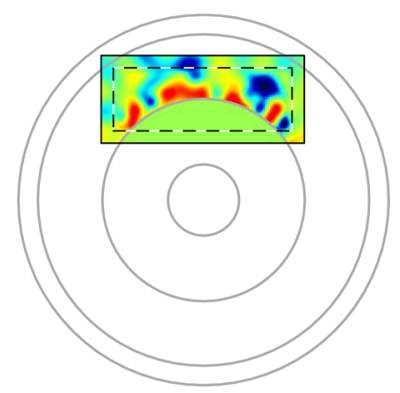

iteration 15

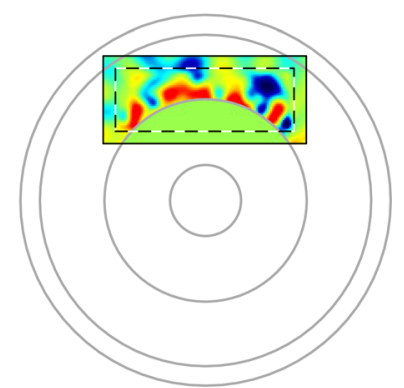

target model

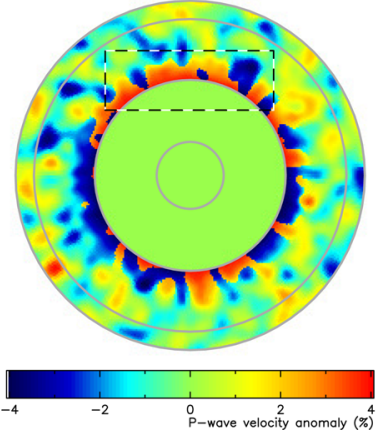

Figure 14. Comparison between a global tomographic inversion and a local tomographic inversion (within the box delimited by the dashed line) in the situation where the velocity structure outside the inversion box is obtained from a prior global inversion with coarse resolution. The reference or starting model employed for the inversion is pictured as Iteration 0 and was obtained by running a global inversion using low frequency data (i.e. with a minimum period of $250 \mathrm{~s}$ ). To build our data set, for 10 seismic sources, we recorded the wavefield at a 50 seismic stations, the seismograms with duration $1600 \mathrm{~s}$ were filtered before the inversion using a low pass filter with cut-off frequency corresponding to a period of $33 \mathrm{~s}$. For the inversion, we used an adjoint conjugate gradient scheme. The maximum resolution allowed by the spatial smoothing of the adjoint gradient varies from $1000 \mathrm{Km}$ at iteration 1 to $300 \mathrm{~km}$ at iteration 15 . After 15 iteration, both the global and the box-tomography models give images that well match the target model pictured in the bottom panel. Both models improved with respect to the model obtained using a 1-D starting model in Fig. 11.

computations (e.g. Cottaar \& Romanowicz 2012). To investigate the use of box tomography for such deep Earth imaging, we conducted synthetic experiments within a 2-D fluid planet. We showed that, when the velocity structure is well known within the planet, box-tomography is faster and more accurate than global tomogra- phy for imaging localized objects. Most importantly, in the delicate situation where the velocity structure is not well constrained within the planet, box-tomography still performs very well and produces accurate images of local structures. We showed that the spatial resolution and the error in the absolute velocities of global tomographic 
(a) global tomography

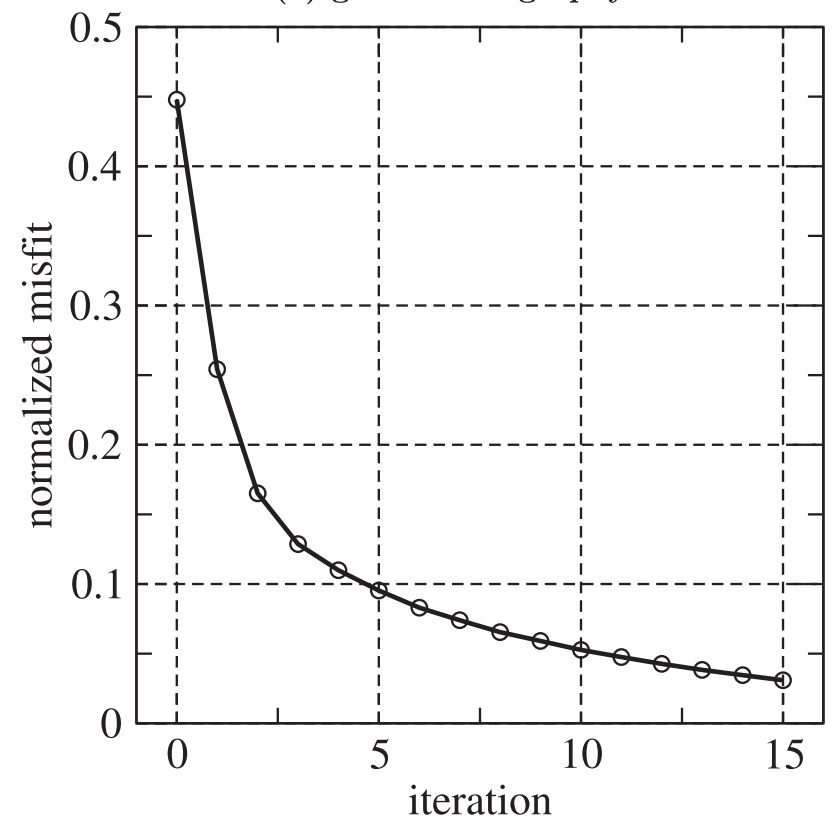

(b) box tomography

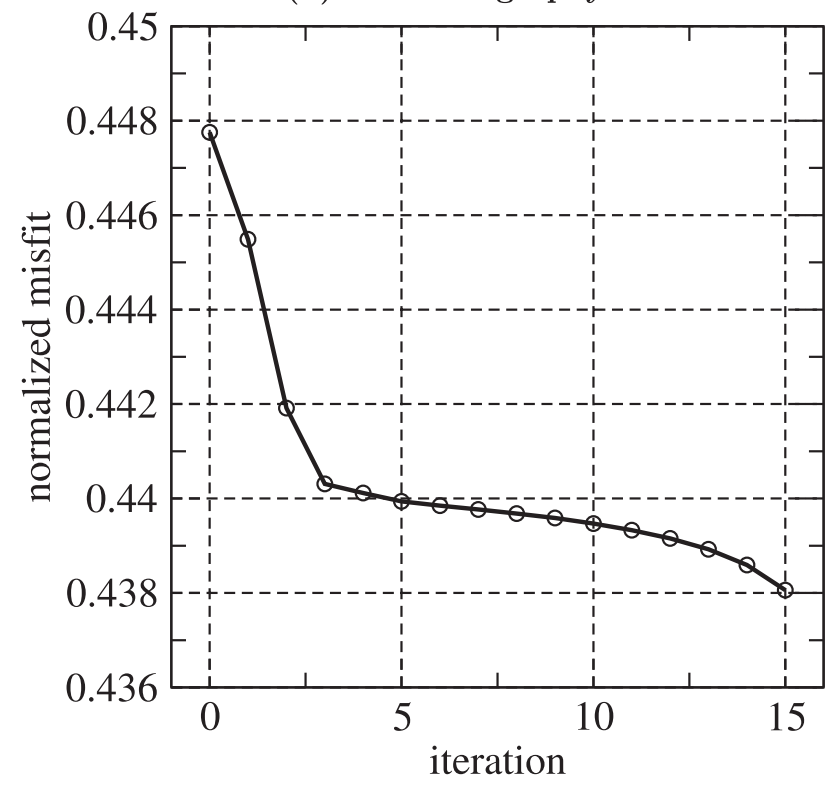

Figure 15. Evolution of the misfit function in eq. (27) as a function of the number of iterations in the situation where the a priori velocity structure outside the imaging box is obtained using a global inversion with coarse resolution. (a) Corresponds to the global inversion pictured in the left panels of Fig. 14. (b) Corresponds to the local inversion pictured in the right panels of Fig. 14.

models can be significantly refined locally using box-tomography. This is important for the geodynamical interpretation of global tomographic models.

Applications to real life problems should not bring any major problems, apart from those inherent to tomographic imaging such as the seismic noise or the limited data coverage. Extension of the box-tomography formalism to the elastic case is straightforward and all aspects of wave propagation (e.g. source properties, attenuation, anisotropy, attenuation, etc.) involved in global seismology can be fully modelled (e.g. Masson \& Romanowicz 2017). Attenuation that (a) global tomography

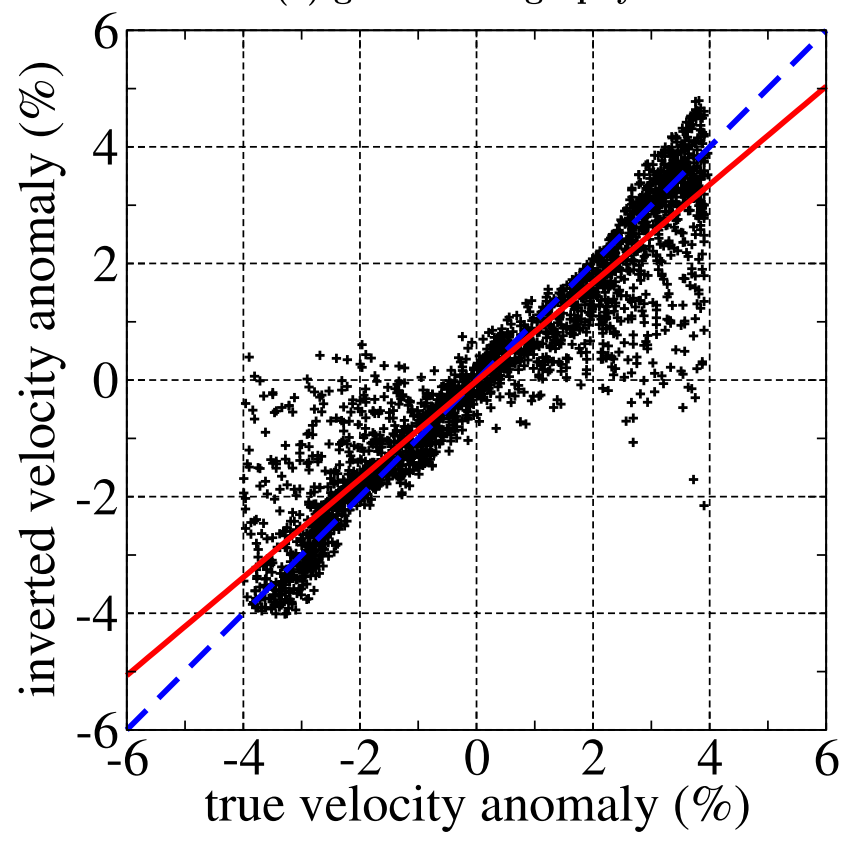

(b) box tomography

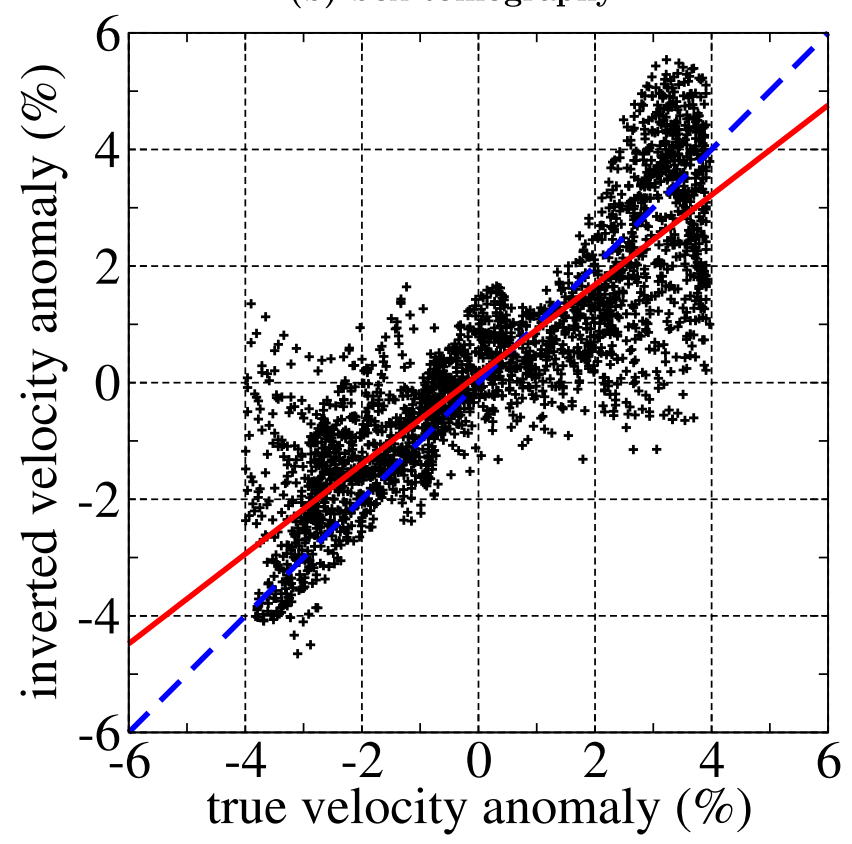

Figure 16. Scatter plots showing the correlation between the inverted velocities and the true velocities in the situation where the a priori velocity structure outside the imaging box is obtained using a global inversion with coarse resolution. The black dots shows the inverted velocities plotted as a function of the true velocities of propagating medium (target model) inside the imaging box delimited by the dashed line in Fig. 8 . The solid red line corresponds to the linear regression of the data. In the ideal situation where the true velocities are recovered by the inversion, all the dots lies on the dashed blue line of identity $y=x$. (a) Corresponds to the velocities obtained with the global inversion pictured in the left panels of Fig. 14. (b) Corresponds to the velocities obtained with the local inversion pictured in the right panels of Fig. 14. 
we did not consider in this study may be problematic in highly dissipative media, nonetheless this is not specific to box tomography and we think our results hold so far the waves that are sensitive to the box region can be recorded at the surface for example ScS, Sdiff and their codas. The computational cost for imaging a remote region is similar to that of a regional inversion (i.e. where all the sources and the receivers are located within the imaged region) plus a limited number of initial global simulations done prior to the inversion. We showed that, when higher order scattering effects are neglected, one can obtain accurate images using only $N_{s}+N_{r}$ initial global simulations, where $N_{s}$ and $N_{r}$ are the number of sources and receivers employed in the inversion, respectively. These initial global simulations may be performed using fast approximate methods (e.g. frequency-wavenumber (FK), Tong et al. 2014a,b; Direct Solution Method (DSM), Monteiller et al. 2015; Spectral element methods for axisymmetric domains (AxiSEM), Nissen-Meyer et al. 2007) together with a 1-D reference model, or, using approximate methods for modelling wave propagation in a 3-D Earth model (e.g. Axisem 3D, Leng et al. 2016), or, using exact wave propagation modelling in a 3-D Earth model (e.g. Komatitsch \& Tromp 1999). When using approximate methods together with a 1-D Earth model, the computational burden is limited and almost negligible compare to that of the local box inversion. When using exact wave propagation modelling in a 3-D Earth model, the computational effort to run the $N_{s}+N_{r}$ initial global simulations becomes significant, nonetheless, it is considerably smaller than that of a global tomographic full waveform inversion (e.g. Bozdağ et al. 2016). Our examples shows that box-tomography can produce accurate images of localized structure using either a 1-D or a 3-D reference Earth model, however, our preference is to start with a 3-D model which gives better results as more information can be gathered from existing tomographic models.

Using a partial implementation of the box-tomography concept with sources outside and stations within the target region and using a 3-D reference model, we have developed a regional elastic model of the North American continent. These results are presented in a separate publication (Clouzet et al., in preparation) and demonstrate the usefulness of the proposed method.

\section{ACKNOWLEDGEMENTS}

We thank two anonymous reviewers and Editor Andrea Morelli for their comments and suggestions. The research leading to these results has been supported through funding from the European Research Council under the European Community's Seventh Framework Programme (FP7-IDEAS-ERC)/ERC Advanced Grant (WAVETOMO). This research used resources of the National Energy Research Scientific Computing Center, a DOE Office of Science User Facility supported by the Office of Science of the U.S. Department of Energy under Contract No. DE-AC02-05CH11231. Barbara Romanowicz acknowledges support from NSF grant EAR1417229 .

\section{REFERENCES}

Aki, K. \& Richards, P.G., 1980. Quantitative Seismology, vol. 1424, Freeman.

Alterman, Z. \& Karal, F., 1968. Propagation of elastic waves in layered media by finite difference methods, Bull. seism. Soc. Am., 58(1), 367-398.

Bamberger, A., Chavent, G., Hemon, C. \& Lailly, P., 1982. Inversion of normal incidence seismograms, Geophysics, 47(5), 757-770.
Baysal, E., Kosloff, D.D. \& Sherwood, J.W., 1983. Reverse time migration, Geophysics, 48(11), 1514-1524.

Bednar, J.B., Bednar, C.J. \& Shin, C.B., 2006. Two-way vs one-way: a case study style comparison, in Proceedings of the SEG Annual Meeting, New Orleans, LA, Society of Exploration Geophysicists.

Belina, F., Ernst, J. \& Holliger, K., 2009. Inversion of crosshole seismic data in heterogeneous environments: comparison of waveform and ray-based approaches, J. Appl. Geophys., 68(1), 85-94.

Berryhill, J.R., 1984. Wave-equation datuming before stack, Geophysics, 49(11), 2064-2066.

Bielak, J. \& Christiano, P., 1984. On the effective seismic input for nonlinear soil-structure interaction systems, Earthq. Eng. Struct. Dyn., 12, $107-119$.

Bielak, J., Loukakis, K., Hisada, Y. \& Yoshimura, C., 2003. Domain reduction method for three-dimensional earthquake modeling in localized regions, Part I: Theory, Bull. seism. Soc. Am., 93(2), 817-824.

Biondi, B. \& Shan, G., 2002. Prestack imaging of overturned reflections by reverse time migration, in SEG Technical Program Expanded Abstracts, vol. 21, pp. 1284-1287.

Borisov, D., Singh, S.C. \& Fuji, N., 2015. An efficient method of 3-D elastic full waveform inversion using a finite-difference injection method for time-lapse imaging, Geophys. J. Int., 202(3), 1908-1922.

Born, M. \& Wolf, E., 1959. Principles of Optics: Electromagnetic Theory of Propagation, Interference and Diffaction of Light, Pergamon Press.

Bouchon, M. \& Sánchez-Sesma, F.J., 2007. Boundary integral equations and boundary elements methods in elastodynamics, Adv. Geophys., 48, 157-189.

Bozdağ, E., Peter, D., Lefebvre, M., Komatitsch, D., Tromp, J., Hill, J., Podhorszki, N. \& Pugmire, D., 2016. Global adjoint tomography: firstgeneration model, Geophys. J. Int., 207(3), 1739-1766.

Broggini, F., Snieder, R. \& Wapenaar, K., 2012. Focusing the wavefield inside an unknown 1D medium: beyond seismic interferometry, Geophysics, 77(5), A25-A28.

Burger, H.R., 1992. Exploration Geophysics of the Shallow Subsurface, Prentice Hall.

Capdeville, Y., To, A. \& Romanowicz, B., 2003. Coupling spectral elements and modes in a spherical earth: an extension to the 'sandwich' case, Geophys. J. Int., 154(1), 44-57.

Capdeville, Y., Stutzmann, É., Wang, N. \& Montagner, J.-P., 2013. Residual homogenization for seismic forward and inverse problems in layered media, Geophys. J. Int., 194(1), 470-487.

Chang, S.-J., Ferreira, A.M., Ritsema, J., Heijst, H.J. \& Woodhouse, J.H., 2015. Joint inversion for global isotropic and radially anisotropic mantle structure including crustal thickness perturbations, J. geophys. Res., 120(6), 4278-4300.

Chen, P., 2011. Full-wave seismic data assimilation: theoretical background and recent advances, Pure appl. Geophys., 168(10), 1527-1552.

Cottaar, S. \& Romanowicz, B., 2012. An unusually large ULVZ at the base of the mantle near Hawaii, Earth planet. Sci. Lett., 355, 213-222.

da Costa Filho, C.A., Ravasi, M., Curtis, A. \& Meles, G.A., 2014. Elastodynamic Green's function retrieval through single-sided Marchenko inverse scattering, Phys. Rev. E, 90(6), doi:10.1103/PhysRevE.90.063201.

Dahlen, F., Hung, S.-H. \& Nolet, G., 2000. Fréchet kernels for finitefrequency traveltimes-I. Theory, Geophys. J. Int., 141(1), 157-174.

De Hoop, A.T., 1958. Representation theorems for the displacement in an elastic solid and their application to elastodynamic diffraction theory, Doctoral thesis, TU Delft.

Dziewonski, A.M. \& Anderson, D.L., 1981. Preliminary reference earth model, Phys. Earth planet. Inter., 25(4), 297-356.

Farmer, P., Miller, D., Pieprzak, A., Rutledge, J. \& Woods, R., 1994. Exploring the subsalt, Leading Edge, 13(8), 837.

Fichtner, A., Bunge, H.-P. \& Igel, H., 2006. The adjoint method in seismology: I. theory, Phys. Earth planet. Inter., 157(1), 86-104.

French, S.W. \& Romanowicz, B.A., 2014. Whole-mantle radially anisotropic shear velocity structure from spectral-element waveform tomography, Geophys. J. Int., 199(3), 1303-1327.

French, S.W. \& Romanowicz, B., 2015. Broad plumes rooted at the base of the earth's mantle beneath major hotspots, Nature, 525(7567), 95-99. 
French, S., Lekic, V. \& Romanowicz, B., 2013. Waveform tomography reveals channeled flow at the base of the oceanic asthenosphere, Science, 342(6155), 227-230.

Godinho, L., Mendes, P.A., Tadeu, A., Cadena-Isaza, A., Smerzini, C., Sánchez-Sesma, F., Madec, R. \& Komatitsch, D., 2009. Numerical simulation of ground rotations along $2 \mathrm{~d}$ topographical profiles under the incidence of elastic plane waves, Bull. seism. Soc. Am., 99(2B), 11471161.

Hashin, Z. \& Shtrikman, S., 1963. A variational approach to the theory of the elastic behaviour of multiphase materials, J. Mech. Phys. Solids, 11(2), 127-140.

Houser, C., Masters, G., Shearer, P. \& Laske, G., 2008. Shear and compressional velocity models of the mantle from cluster analysis of long-period waveforms, Geophys. J. Int., 174(1), 195-212.

Klimeš, L., 2002. Correlation functions of random media, Pure appl. geophys., 159(7-8), 1811-1831.

Komatitsch, D. \& Tromp, J., 1999. Introduction to the spectral element method for three-dimensional seismic wave propagation, Geophys. J. Int., 139(3), 806-822.

Krishnan, S., Ji, C., Komatitsch, D. \& Tromp, J., 2006. Case studies of damage to tall steel moment-frame buildings in southern California during large San Andreas earthquakes, Bull. seism. Soc. Am., 96(4A), 15231537.

Kustowski, B., Ekström, G. \& Dziewoński, A., 2008. Anisotropic shearwave velocity structure of the Earth's mantle: a global model, J. geophys. Res., 113(B6), doi:10.1029/2007JB005169.

Lekić, V. \& Romanowicz, B., 2011. Inferring upper-mantle structure by full waveform tomography with the spectral element method, Geophys. J. Int., 185(2), 799-831.

Leng, K., Nissen-Meyer, T. \& van Driel, M., 2016. Efficient global wave propagation adapted to 3-D structural complexity: a pseudospectral/spectral-element approach, Geophys. J. Int., 207(3), $1700-1721$.

Li, X.D. \& Romanowicz, B., 1995. Comparison of global waveform inversions with and without considering cross-branch modal coupling, Geophys. J. Int., 121(3), 695-709.

Li, X.-D. \& Romanowicz, B., 1996. Global mantle shear velocity model developed using nonlinear asymptotic coupling theory, J. geophys. Res., 101(B10), 22 245-22 272.

Masson, Y. \& Pride, S.R., 2015. Mapping the mechanical properties of rocks using automated microindentation tests, J. geophys. Res., 120(10), 7138-7155.

Masson, Y. \& Romanowicz, B., 2017. Fast computation of synthetic seismograms within a medium containing remote localized perturbations: a numerical solution to the scattering problem, Geophys. J. Int., 208(2), 674-692

Masson, Y., Cupillard, P., Capdeville, Y. \& Romanowicz, B., 2014. On the numerical implementation of time-reversal mirrors for tomographic imaging, Geophys. J. Int., 196, 1580-1599.

Mégnin, C. \& Romanowicz, B., 2000. The three-dimensional shear velocity structure of the mantle from the inversion of body, surface and highermode waveforms, Geophys. J. Int., 143(3), 709-728.

Meschede, M. \& Romanowicz, B., 2015. Lateral heterogeneity scales in regional and global upper mantle shear velocity models, Geophys. J. Int., 200(2), 1078-1095.

Moczo, P., Bystrický, E., Kristek, J., Carcione, J.M. \& Bouchon, M., 1997. Hybrid modeling of $P-S V$ seismic motion at inhomogeneous viscoelastic topographic structures, Bull. seism. Soc. Am., 87(5), $1305-1323$.

Monteiller, V., Chevrot, S., Komatitsch, D. \& Fuji, N., 2013. A hybrid method to compute short-period synthetic seismograms of teleseismic body waves in a 3-D regional model, Geophys. J. Int., 192(1), 230-247.

Monteiller, V., Chevrot, S., Komatitsch, D. \& Wang, Y., 2015. Threedimensional full waveform inversion of short-period teleseismic wavefields based upon the SEM-DSM hybrid method, Geophys. J. Int., 202(2), 811-827.

Mulder, W.A. \& Plessix, R.-E., 2004. A comparison between one-way and two-way wave-equation migration, Geophysics, 69(6), 1491-1504.
Nissen-Meyer, T., Fournier, A. \& Dahlen, F.A., 2007. A two-dimensional spectral-element method for computing spherical-earth seismograms-I. Moment-tensor source, Geophys. J. Int., 168(3), 1067-1092.

Opršal, I., Matyska, C. \& Irikura, K., 2009. The source-box wave propagation hybrid methods: general formulation and implementation, Geophys. J. Int., 176(2), 555-564.

Pageot, D., Operto, S., Vallée, M., Brossier, R. \& Virieux, J., 2013. A parametric analysis of two-dimensional elastic full waveform inversion of teleseismic data for lithospheric imaging, Geophys. J. Int., 193(3), $1479-1505$.

Pica, A., Diet, J. \& Tarantola, A., 1990. Nonlinear inversion of seismic reflection data in a laterally invariant medium, Geophysics, 55(3), 284292.

Plessix, R.-E., 2006. A review of the adjoint-state method for computing the gradient of a functional with geophysical applications, Geophys. J. Int., 167(2), 495-503.

Polak, E. \& Ribiere, G., 1969. Note sur la convergence de méthodes de directions conjuguées, Revue française d'informatique et de recherche opérationnelle, série rouge, 3(1), 35-43.

Prothero, W., Taylor, W.J. \& Eickemeyer, J., 1988. A fast, two-point, threedimensional raytracing algorithm using a simple step search method, Bull. seism. Soc. Am., 78(3), 1190-1198.

Ritsema, J., Deuss, A., Van Heijst, H. \& Woodhouse, J., 2011. S40RTS: a degree-40 shear-velocity model for the mantle from new Rayleigh wave dispersion, teleseismic traveltime and normal-mode splitting function measurements, Geophys. J. Int., 184(3), 1223-1236.

Robertsson, J.O.A. \& Chapman, C.H., 2000. An efficient method for calculating finite-difference seismograms after model alterations, Geophysics, 65(3), 907-918.

Robertsson, J.O.A., Ryan-Grigor, S., Sayers, C.M. \& Chapman, C.H., 2000. A finite-difference injection approach to modeling seismic fluid flow monitoring, Geophysics, 65(3), 896-906.

Roecker, S., Baker, B. \& McLaughlin, J., 2010. A finite-difference algorithm for full waveform teleseismic tomography, Geophys. J. Int., 181(2), 10171040.

Rose, J.H., 2002. 'single-sided' autofocusing of sound in layered materials, Inverse Probl., 18(6), 1923-1934.

Schuster, G.T., 2009. Seismic Interferometry, vol. 1, Cambridge Univ. Press.

Simmons, N.A., Forte, A.M., Boschi, L. \& Grand, S.P., 2010. GyPSuM: a joint tomographic model of mantle density and seismic wave speeds, J. geophys. Res., 115, B12310, doi:10.1029/2010JB007631.

Snieder, R., 2002. General theory of elastic wave scattering, in Scattering and Inverse Scattering in Pure and Applied Science, chap. 1.7.1, pp. 528-542, eds Pike, R. \& Sabatier, P., Academic Press.

Snieder, R. \& Sambridge, M., 1992. Ray perturbation theory for traveltimes and ray paths in 3-D heterogeneous media, Geophys. J. Int., 109(2), 294322.

Su, W.J. \& Dziewonski, A.M., 1997. Simultaneous inversion for 3-D variations in shear and bulk velocity in the mantle, Phys. Earth planet. Inter, 100(1-4), 135-156.

Taflove, A. \& Brodwin, M.E., 1975. Numerical solution of steady-state electromagnetic scattering problems using the time-dependent Maxwell's equations, IEEE Trans. Microw. Theory Tech., 23(8), 623-630.

Tarantola, A., 1984. Inversion of seismic reflection data in the acoustic approximation, Geophysics, 49(8), 1259-1266.

Tarantola, A., 1988. Theoretical background for the inversion of seismic waveforms including elasticity and attenuation, Pure appl. Geophys., 128(1-2), 365-399.

Tarantola, A. \& Valette, B., 1982. Generalized nonlinear inverse problems solved using the least squares criterion, Rev. Geophys., 20(2), 219-232.

To, A., Romanowicz, B., Capdeville, Y. \& Takeuchi, N., 2005. 3D effects of sharp boundaries at the borders of the African and Pacific Superplumes: observation and modeling, Earth planet. Sci. Lett., 233(1), 137-153.

Tong, P., Chen, C.-w., Komatitsch, D., Basini, P. \& Liu, Q., 2014a. Highresolution seismic array imaging based on an SEM-FK hybrid method, Geophys. J. Int., 197(1), 369-395.

Tong, P., Komatitsch, D., Tseng, T.-L., Hung, S.-H., Chen, C.-W., Basini, P. \& Liu, Q., 2014b. A 3-D spectral-element and frequency-wave number 
hybrid method for high-resolution seismic array imaging, Geophys. Res. Lett., 41(20), 7025-7034.

Tromp, J., Tape, C. \& Liu, Q., 2005. Seismic tomography, adjoint methods, time reversal and banana-doughnut kernels, Geophys. J. Int., 160(1), 195216.

van Manen, D.-J., Robertsson, J.O. \& Curtis, A., 2007. Exact wave field simulation for finite-volume scattering problems, J. acoust. Soc. Am., 122(4), EL115-EL121.

Virieux, J. \& Operto, S., 2009. An overview of full-waveform inversion in exploration geophysics, Geophysics, 74(6), WCC1-WCC26.

Wapenaar, K., 2014. Single-sided Marchenko focusing of compressional and shear waves, Phys. Rev. E, 90(6), doi:10.1103/PhysRevE.90.063202.

Wapenaar, K., Slob, E. \& Snieder, R., 2008. Seismic and electromagnetic controlled-source interferometry in dissipative media, Geophys. Prospect., 56(3), 419-434.

Wapenaar, K., Broggini, F., Slob, E. \& Snieder, R., 2013. Three-dimensional single-sided Marchenko inverse scattering, data-driven focusing, Green's function retrieval, and their mutual relations, Phys. Rev. Lett., 110(8), doi:10.1103/PhysRevLett.110.084301.

Wen, L. \& Helmberger, D.V., 1998. A two-dimensional P-SV hybrid method and its application to modeling localized structures near the core-mantle boundary, J. geophys. Res., 103(B8), 17 901-17 918.
Whitmore, N., 1983. Iterative depth migration by backward time propagation, in Proceedings of the SEG Annual Meeting, Las Vegas, NV, Society of Exploration Geophysicists.

Willemsen, B., Malcolm, A. \& Lewis, W., 2016. A numerically exact local solver applied to salt boundary inversion in seismic full-waveform inversion, Geophys. J. Int., 204(3), 1703-1720.

Woodhouse, J.H. \& Dziewonski, A.M., 1984. Mapping the upper mantle: three-dimensional modeling of Earth structure by inversion of seismic waveforms, J. Geophys. Res., 89(B7), 5953-5986.

Yoon, K., Shin, C., Suh, S., Lines, L.R. \& Hong, S., 2003. 3D reversetime migration using the acoustic wave equation: an experience with the SEG/EAGE data set, Leading Edge, 22(1), 38-41.

Yoon, K., Marfurt, K.J. \& Starr, W., 2004. Challenges in reverse-time migration, in Proceedings of the SEG Annual Meeting, Denver, CO, Society of Exploration Geophysicists.

Yoshimura, C., Bielak, J., Hisada, Y. \& Fernández, A., 2003. Domain reduction method for three-dimensional earthquake modeling in localized regions, Part II: Verification and applications, Bull. seism. Soc. Am., 93(2), 825-841.

Zhao, L., Wen, L., Chen, L. \& Zheng, T., 2008. A two-dimensional hybrid method for modeling seismic wave propagation in anisotropic media, $J$. geophys. Res., 113, B12307, doi:10.1029/2008JB005733. 